\title{
Non-Canonical Targets of HIF1a Drive Cell-Type-Specific Dysfunction
}

Kevin C. Allan ${ }^{1}$, Lucille R. Hu${ }^{1}$, Andrew R. Morton¹, Marissa A. Scavuzzo1, Artur S. Gevorgyan ${ }^{1}$, Benjamin L.L. Clayton ${ }^{1}$, Ilya R. Bederman ${ }^{1}$, Stevephen Hung ${ }^{1}$ Cynthia F. Bartels ${ }^{1}$, Mayur Madhavan ${ }^{1}$, Paul J. Tesar ${ }^{1^{*}}$

${ }^{1}$ Department of Genetics and Genome Sciences, Case Western Reserve University School of Medicine, Cleveland, Ohio 44106, USA.

*Correspondence: paul.tesar@case.edu

\section{SUMMARY}

All mammalian cells sense and respond to insufficient oxygen, or hypoxia, through the activity of hypoxia-inducible factors (HIFs), an evolutionarily conserved family of transcriptional regulators that promote oxygen-independent energy metabolism and angiogenesis. While HIF activation is transiently protective for all cells, prolonged HIF activity drives distinct pathological responses in different tissues. How HIF achieves this pleiotropic effect is largely unknown. Here, we demonstrate that non-canonical targets of HIF1a impair the function of oligodendrocyte progenitor cells (OPCs) to generate oligodendrocytes. Beyond the canonical gene targets shared between all cell types, HIF1a also bound to and activated a unique set of targets in OPCs including Asc/2 and D/x3. Each of these targets, when ectopically expressed, was sufficient to block oligodendrocyte development through suppression of the key oligodendrocyte regulator Sox10. Chemical screening revealed that inhibition of MEK/ERK signaling overcame the HIF1a-mediated block in oligodendrocyte generation by restoring Sox10 expression without impacting canonical HIF1a activity. Collectively this work defines the mechanism by which chronic HIF1a suppresses oligodendrocyte formation. More broadly, we establish that cell-type-specific HIF1a targets, independent of the canonical hypoxia response, perturb cell function and drive disease in chronic hypoxia. 


\section{INTRODUCTION}

The ability to sense and respond to fluctuations in oxygen levels is required to maintain homeostasis in every cell in the body (Kaelin and Ratcliffe, 2008; Semenza, 2012). Insufficient concentrations of molecular oxygen rapidly trigger an evolutionary conserved transcriptional response that enables cell survival in low oxygen by promoting anaerobic metabolism for energy production as well as angiogenesis and erythropoiesis to increase access to local oxygen. While this is initially protective, prolonged activation of this response leads to cellular dysfunction and disease in many tissues. For example, the response to chronic hypoxia blocks white matter formation in premature birth (Scafidi et al., 2014; Volpe, 2009; Volpe et al., 2011), promotes inflammation and insulin resistance in obesity (Lee et al., 2014), and impairs hematopoietic stem cell transplantation capacity (Takubo et al., 2010). This cellular dysfunction has largely been attributed to prolonged activation of the canonical response to low oxygen shared across all cell types; however, it is difficult to explain how activation of a conserved set of hypoxia signature genes can lead to such diverse cellular phenotypes. An alternative unexplored possibility is that cell-type-specific differences in chromatin landscape enable access to unique non-canonical targets, which could account for tissue-specific pathologies.

The response to low oxygen is mediated by hypoxia inducible factors (HIFs), a family of transcription factors that are stabilized under hypoxic conditions in all mammalian cells and are primarily thought to upregulate multiple pathways that adapt cells to low oxygen (Cassavaugh and Lounsbury, 2011; Choudhry and Harris, 2018; Kupferschmidt, 2019). HIFs are heterodimeric complexes consisting of an alpha and beta subunit. In the presence of oxygen, alpha subunits are hydroxylated by prolyl-hydroxylases, allowing for recognition and ubiquitination by von Hippel Lindau (VHL) (Ivan et al., 2001; Jaakkola et al., 2001), and rapid degradation by the proteasome. However, in low oxygen conditions the alpha subunits escape hydroxylation, avoid degradation, and translocate to the nucleus to pair with constitutive beta subunits and regulate gene expression (Cassavaugh and Lounsbury, 2011; Choudhry and Harris, 2018; Semenza, 2007). The HIF1a 
motif is present more than 1 million times in the genome; however, HIF1a binds to only a small fraction of these sites, which suggests that HIF1a binding is heavily regulated (Schodel et al., 2011; Smythies et al., 2019). Still, the determinants of HIF1a binding in each cell type and whether cell-type-specific targets are functional remain unknown.

The central nervous system (CNS) consumes $20 \%$ of the body's oxygen supply and white matter of the CNS is highly susceptible to hypoxic insults as seen in stroke, vascular dementia, respiratory distress syndromes, premature birth, and subsets of cerebral palsy (Hankey, 2017; Salmaso et al., 2014; Shindo et al., 2016; Volpe, 2009). In fact, chronic HIF1a activity is sufficient to block white matter development (Yuen et al., 2014). White matter of the CNS is formed by oligodendrocytes, which wrap neuronal axons in a lipid-rich protective sheath called myelin, allowing for rapid transmission of action potentials and maintenance of axonal integrity (Chang et al., 2016; Emery, 2010; Nave, 2010). Oligodendrocytes arise from oligodendrocyte progenitor cells (OPCs), which are prevalent in the developing and adult CNS, and HIF1a accumulation has been shown to be sufficient to impair oligodendrocyte formation from OPCs (Jablonska et al., 2016; van Tilborg et al., 2018; Yuen et al., 2014). However, the mechanism of the HIF1a-mediated block in oligodendrocyte formation from OPCs remains unclear. In this study, we use OPCs as an archetypal hypoxia-disease relevant cell type to define the mechanism by which chronic HIF1a drives cell dysfunction.

\section{RESULTS}

\section{Knockout of VHL models chronic HIF1a accumulation in iPSC-derived OPCs}

Defining the mechanisms by which HIF activity perturbs cell function is notoriously challenging as HIFs are rapidly degraded, in minutes, when cells are restored to normoxia. Because of this instability and the low abundance of HIFs, biochemical studies often require extraordinary numbers of cells, which is challenging for hypoxia disease relevant cell types outside of cancer cell lines. To explore the mechanisms underlying the HIF-mediated block in oligodendrocyte 
development from OPCs, we generated a cellular model of chronic HIF1a accumulation in mouse pluripotent stem cell-derived OPCs, which are uniquely scalable and amenable to genetic manipulation (Hubler et al., 2018; Lager et al., 2018; Najm et al., 2015; Najm et al., 2011). CRISPR-Cas9-mediated knockout of VHL, a central component of the ubiquitin-proteasome system that degrades HIFs (Choudhry and Harris, 2018; Haase, 2009; Rechsteiner et al., 2011), in OPC cultures resulted in stable HIF1a protein accumulation and significant 9-fold and 18-fold activation of downstream hallmark HIF1a targets Vegfa and Bnip3, respectively (Figures 1A, 1B, S1A and S1B). The response of OPCs to VHL knockout mirrored that of OPCs cultured in hypoxia $\left(1 \% \mathrm{O}_{2}\right)$, which led to a significant 11-fold increase in Vegfa and 26-fold increase in Bnip3 (Figures 1C and 1D). VHL knockout OPCs were generated with two independent single guide RNAs targeting $\mathrm{Vhl}$ (sgVhl and sgVhl.2), each of which caused significant decreases in Vhl transcript and protein levels through Cas9-mediated insertion-deletions (in-dels) at the respective target sites compared to control (Cas9 expressing OPCs with no sgRNA) (Figures S1C-S1E). sgVhl OPCs were used for a majority of the data in the study; however, we confirmed key findings in sgVhl.2 OPCs, OPCs treated with physiological hypoxia, primary mouse OPCs exposed to hypoxia, and human OPCs in pluripotent stem cell-derived oligocortical spheroids (Madhavan et al., 2018).

\section{HIF1a accumulation specifically delays OPC differentiation into oligodendrocytes}

HIF1a accumulation in OPCs is sufficient to impair oligodendrocyte formation (Yuen et al., 2014). To test whether HIF1a accumulation is a general or specific inhibitor of OPC differentiation, sgVhl OPCs were stimulated to form either astrocytes (Grinspan et al., 2000), or oligodendrocytes (Baas et al., 1997; Barres et al., 1994; Gao et al., 1998; Najm et al., 2011) (Figure 1E). This revealed a significant 4-fold reduction in oligodendrocyte formation by staining for myelin basic protein (MBP), a marker of mature oligodendrocytes, with no change in astrocyte formation by staining for glial fibrillary acidic protein (GFAP) in sgVhl OPCs compared to control (Figures 1E-1G, and 
S1F). This suggests HIF1a specifically blocks oligodendrocyte formation from OPCs; however, at what stage HIF1a accumulation impairs the formation of oligodendrocytes is unknown. Staining for early $(\mathrm{O} 4+)$, intermediate $(\mathrm{O} 1+)$, and late $(\mathrm{MBP}+)$ oligodendrocyte markers throughout the differentiation process demonstrated a significant and delayed acquisition of all oligodendrocyte markers in sgVhl and sgVhl.2 OPCs compared to control (Figures $1 \mathrm{H}-1 \mathrm{~L}$, and S1G-S1I). These data suggest that HIF1a accumulation impairs early OPC differentiation, thereby delaying the formation of oligodendrocytes and ultimately myelin.

\section{HIF1a binds proximal to promoters and indirectly suppresses Sox10 expression in OPCs}

To delineate the gene targets of HIF1a in OPCs responsible for blocking oligodendrocyte development, chromatin-linked immunoprecipitation sequencing (ChIP-seq) was used to map its genome-wide chromatin binding profile. Utilizing 100 million control and sgVhl OPCs for HIF1a ChIP-seq identified 503 high-stringent peaks (FDR<0.001) in sgVhl OPCs with clear enrichment proximal to the annotated transcription start site (TSS), which agrees with HIF1a as a promoter centric transcription factor (Schodel et al., 2011; Smythies et al., 2019) (Figures 2A and S2A). HIF1a was enriched at canonical target genes Vegfa and Bnip3 and globally peaks were enriched for HIF motifs and motifs of transcription factors that have been shown to interact with HIF1a including Sp1, c-Myc, and Bmal1 (Huang, 2008; Kaluz et al., 2003; Wu et al., 2017) (Figures S2A and S2B). HIF1a was not found proximal to Sirt1, Wnt7a, or Wnt7b (Figures S2C-S2E), which have previously been suggested as putative HIF effectors in OPCs (Jablonska et al., 2016; Yuen et al., 2014). Moreover, neither sgVhl OPCs nor wild type OPCs exposed to hypoxia exhibited increased expression of Sirt1, Wnt7a, or Wnt7b transcripts, suggesting that other targets are likely functioning to block oligodendrocyte formation (Figures S2F-S2I) (Zhang et al., 2020).

To determine the functional targets of HIF1a, we performed RNA-seq of sgVhl and control OPCs. Overlapping transcripts that significantly changed between sgVhl and control OPCs (Padj<0.05) with direct targets of HIF1a revealed that HIF1a directly bound to 61 significantly 
increased genes and only 1 significantly decreased gene (Figure 2B), consistent with the role of HIF1a as a transcriptional activator (Dengler et al., 2014; Guimaraes-Camboa et al., 2015). ChIPseq for a marker of active chromatin, H3K27Ac (Creyghton et al., 2010), in sgVhl and control OPCs mirrored these findings with a greater number of regions exhibiting a significant gain of H3K27Ac in sgVhl OPCs compared to control $(F D R<0.1)$, and these regions were enriched for HIF motifs (Figures S2J and S2K). To define the top functional targets of HIF1a in OPCs, we overlapped direct HIF1a targets (FDR<0.001) with genes that exhibited both increased transcription (P-adj<0.05) and increased H3K27Ac (FDR<0.1) in sgVhl OPCs compared to control (Figure 2C). Hits were independently validated in OPCs treated with hypoxia, which showed that all of our top 10 HIF1a targets were significantly upregulated compared to normoxia (Figures 1D and 2D).

Of note, HIF1a did not bind proximal to any master regulators of oligodendrocyte development, such as Sox10, Olig2, and Nkx2.2. However, the proximal promoter region of Sox10, a basic helix loop helix (bHLH) transcription factor required for formation of oligodendrocytes from OPCs (Stolt et al., 2004; Stolt et al., 2002), showed a reduction of H3K27Ac in sgVhl OPCs (Figure 2E). The decrease in H3K27Ac correlated with a reduction of Sox10 mRNA and protein as well as reduction in expression of downstream Sox10 target genes, Plp1 and Pdgfra, in sgVhl OPCs and primary OPCs treated with hypoxia $\left(1 \% \mathrm{O}_{2}\right)($ Figures $2 \mathrm{~F}, 2 \mathrm{G}$, and S2LS2N). These data suggest that HIF1a activates gene targets that may ultimately impair expression of Sox10 to block oligodendrocyte development.

\section{Chromatin accessibility and cell-type-specific transcription factors define non-canonical HIF1a targets}

Whether sustained activation of unique cell-type-specific HIF1a target genes drive cellular dysfunction in hypoxia is unknown. To categorize canonical and cell-type-specific targets of HIF1a, we overlapped HIF1a targets in OPCs with HIF1a targets from the limited number of 
publicly available datasets derived from other mouse cell types including melanocytes (Loftus et al., 2017), T-cells (Ciofani et al., 2012), and embryonic heart (Guimaraes-Camboa et al., 2015). This analysis identified 51 genes that were HIF1a targets across all 4 cell types ("HIF1a core targets"), 152 genes that were HIF1a targets only in OPCs based on these datasets ("OPCspecific HIF1a targets") and 2250 genes that were specific to either heart, T-cells, or melanocytes ("Other tissue-specific HIF1a targets") (Figure 3A). Both core and OPC-specific HIF1a target genes collectively increased in expression in sgVhl OPCs compared to control, and both of these gene sets showed a significantly greater increase in expression compared to the other tissue specific HIF1a targets in sgVhl OPCs (Figures 3B and S3A).

Gene ontology (GO) analysis of core HIF1a target genes showed enrichment for metabolic and hypoxia pathways, which agrees with HIF1a's canonical role to promote glycolysis in a majority of cell types (Figure 3C) (Choudhry and Harris, 2018; Majmundar et al., 2010; Miska et al., 2019; Nagao et al., 2019). In fact, more than half of the enzymes in the glycolysis pathway were direct HIF1a targets in OPCs, and both sgVhl and sgVhl.2 OPCs exhibited a 2-fold increase in levels of the glycolysis byproduct, lactate, compared to control OPCs (Figures 3D and 3E). Interestingly, GO analysis for cell-type-specific HIF1a targets demonstrated enrichment for pathways separate from the canonical hypoxic response and related to the tissue of origin (Figure S3B). In particular, GO analysis for OPC-specific HIF1a targets showed enrichment for neural development and differentiation pathways (Figure 3C).

To better understand the determinants of the cell type specificity of HIF1a binding profiles, we compared the chromatin landscape in OPCs at core, OPC-specific, and other tissue-specific HIF1a peaks (Figure S3C). The core and OPC-specific HIF1a peaks exhibited a greater enrichment for HIF1a, H3K27Ac and open chromatin (defined by ATAC-seq regions in nontransduced OPCs) compared to other tissue-specific HIF1a sites (Figure 3F). This agrees with previous findings that HIF1a preferentially binds open and active chromatin (Smythies et al., 2019; Xia and Kung, 2009). However, there was a subset of other tissue specific HIF1a peaks enriched 
for open and active chromatin that lacked HIF1a binding in OPCs, suggesting that chromatin accessibility and activity were not the sole predictors of HIF1a binding. Motif enrichment analysis under cell-type-specific HIF1a peaks demonstrated an enrichment for lineage defining transcription factors such as the Mitf family in melanocytes (Levy et al., 2006), Nkx2 family in embryonic heart (Bartlett et al., 2010), and basic-helix-loop helix (bHLH) motifs in OPCs (Figure S3D). Specifically, the motif for Olig2, a lineage-defining bHLH transcription factor in OPCs (Yu et al., 2013), was highly enriched (in the top 5\% of motifs) under OPC-specific HIF1a peaks, whereas the Olig2 motif was not enriched under any other tissue-specific HIF1a peaks (Figure S3D). Collectively, these data suggest that, outside of canonical HIF1a targets, chromatin accessibility and interaction with lineage defining transcription factors determine HIF1a's unique non-canonical targets in each cell type.

\section{Cell-type-specific targets of HIF1a suppress oligodendrocyte formation and Sox10}

Out of the top 10 targets of HIF1a in OPCs, Ascl2 and DIx3 were the only OPC-specific HIF1a targets (Figure 2C). Both are transcription factors that regulate differentiation of somatic stem cells in the periphery and are not normally expressed by any cell type in the mouse CNS (Tabula Muris et al., 2018; Zhang et al., 2014); however, both accumulate at the protein level in sgVhl OPCs compared to control (Figures S3E-S3G). This is reflected by the lack of active chromatin at $A s c / 2$ and D/x3 loci in control OPCs; however, both demonstrate robust HIF1a peaks proximal to their promoters specifically in sgVhl OPCs compared to heart, melanocytes and T-cells along with gained H3K27Ac in sgVhl OPCs compared to control OPCs (Figure 3G). Activation of both $A s c / 2$ and $D / x 3$ transcripts also validated in primary OPCs treated with hypoxia $\left(1 \% \mathrm{O}_{2}\right)$ compared to normoxia (Figure S3H). In fact, ASCL2 was induced in vivo in the brains of mouse pups reared in chronic hypoxia $\left(10 \% \mathrm{O}_{2}\right)$ and this correlated with a decrease in white matter proteins MBP and MAG compared to normoxia reared controls (Figures S3I and S3J). 

technology was sufficient to impair the acquisition of early $(\mathrm{O} 4+)$, intermediate $(\mathrm{O} 1+)$, and late $(\mathrm{MBP}+)$ oligodendrocyte markers across the course of differentiation (Figure $3 \mathrm{H}$ ). Moreover, ectopic expression of DIx3 and Ascl2 also led to a significant reduction in Sox10 expression (Figures $3 \mathrm{I}$ and S3K), whereas activation of shared HIF1a core targets, Slc16a3 and Vegfa, did not (Figures S3K and S3L). These data demonstrate that non-canonical HIF1a targets are sufficient to impair oligodendrocyte formation and emphasize that cell-type-specific targets of HIF1a play a role in driving cellular dysfunction.

\section{Chemical inhibition of MEK/ERK increases oligodendrocyte formation from sgVhI OPCs}

To identify potential pathways that could overcome the HIF1a-indcued cellular pathology of OPCs, we tested a library of 1753 bioactive compounds for the ability to increase the formation of MBP+ oligodendrocytes relative to DMSO treated sgVhl OPCs, which exhibited a consistent differentiation deficit compared to DMSO treated control OPCs (Figures 4A and S4A-S4D). Compounds that were non-toxic (fold change in total cell number $>0.7$ relative to DMSO treated sgVhl OPCs) and enhanced the number and percentage of MBP+ oligodendrocytes (fold change $>3$ relative to DMSO treated sgVhl OPCs) were considered primary hits (Figure S4E). MEK inhibitors were enriched among the primary hits and, as a class, demonstrated a significant increase in oligodendrocyte formation compared to all other non-toxic compounds tested in the primary screen (Figures 4B, 4C, and S4F). Interestingly, drugs previously identified to enhance oligodendrocyte formation, such as miconazole and clemastine (Mei et al., 2014; Najm et al., 2015), were not enriched as hits in this screen, highlighting the ability of this screen to identify context-specific modulators of the differentiation block imposed by HIF1a.

To identify compounds that were effective across a range of doses, we performed an 8point dose curve from $10 \mu \mathrm{M}$ to $78 \mathrm{nM}$ of the top 14 hits. Performing unbiased hierarchical clustering of the results revealed that all 5 MEK inhibitors clustered together and led to a 
pronounced increase in oligodendrocyte formation relative to DMSO treated sgVhl OPCs, with AZD8330 and PD0325091 outperforming PD318088 and Selumetinib (Figures 4D, 4E, and S4G). The ability of these different MEK inhibitors to increase oligodendrocyte formation correlated with their on-target IC50 values for MEK1 and MEK2 as well as their on-target ability to reduce ERK1/2 phosphorylation in sgVhl OPCs (Figures 4F, 4G, and S4H). Performance of an 8-point dose curve consisting of 12 drugs that each inhibit a potential downstream target of MEK revealed that ERK1/2 inhibitors led to the greatest increase in oligodendrocyte formation compared to the other classes of drugs tested (Shaul and Seger, 2007; Yohe et al., 2018) (Figures S4I and S4J). Collectively, these results demonstrate that chemical inhibition of MEK/ERK acts as a node of intervention to increase the formation of oligodendrocytes in the context of HIF1a accumulation.

\section{MEK/ERK inhibition drives Sox10 expression without changing HIF1a activity}

We next asked whether these drugs increased differentiation of sgVhl OPCs by inhibiting HIF1a signaling or circumventing HIF1a by driving genes critical for oligodendrocyte development. Treating OPCs with 300nM AZD8330, our most potent MEK inhibitor (Figures 4F and 4G), for 14 hours led to no change in HIF1a translocation to the nucleus compared to DMSO treated sgVhl OPCs (Figure 5A). Performing RNA-seq on control and sgVhl OPCs treated with either DMSO or 300nM AZD8330 for 14 hours mirrored these results and demonstrated that AZD8330 treatment did not change HIF1a target gene expression, such as $A s c / 2$ and D/x3, in sgVhl OPCs compared to DMSO (Figures 5B, 5C, and S5A). This demonstrates that MEK inhibitor treatment does not directly counter HIF1a activity, but rather circumvents the effect of HIF1a accumulation by increasing oligodendrocyte differentiation despite persistent HIF signaling.

Gene set enrichment analysis (GSEA) revealed that AZD8330 treatment of sgVhl OPCs led to an enrichment for "Oligodendrocyte Differentiation" and "Oligodendrocyte Development" pathways, which were normally depleted in sgVhl OPCs compared to control (Figures 5D and 5E). Further supporting this, AZD8330 treatment of sgVhl OPCs led to a significant increase in 
the subset of genes within the GO term “Oligodendrocyte Development, GO:0014003" that were normally decreased in sgVhl OPCs compared to control (fold change of sgVhl to control OPCs $<0.75$ ), such as Sox10 and Myrf (Figures 5F and 5G). We validated the AZD8330-mediated increase in expression of both of these transcription factors, which are critical for oligodendrocyte differentiation, by qPCR (Figure 5G). To confirm that this was a function of impaired MEK/ERK signaling, treatment of sgVhl OPCs with ERK1/2 inhibitors AZD0364 and SCH772984 led to a similar increase in Sox10 expression compared to DMSO (Figure S5B). Collectively, these data suggest that the reduction of Sox10 expression by cell-type-specific HIF1a targets is critical for the HIF-mediated block in oligodendrocyte differentiation, such that restoration of Sox10 expression without altering canonical HIF function restores oligodendrocyte formation.

\section{MEK/ERK inhibition drives oligodendrocyte formation in hypoxic regions of human}

\section{oligocortical spheroids}

In order to ascertain if these mechanisms are conserved in the human context, we leveraged a pluripotent stem cells (Madhavan et al., 2018). The interior of human brain spheroids is hypoxic (Brawner et al., 2017; Giandomenico and Lancaster, 2017), and we hypothesized that these hypoxic regions would inhibit oligodendrocyte formation, which could be overcome using a MEK inhibitor. To test this, we treated oligocortical spheroids with either DMSO or 300nM AZD8330 for 4 days starting at 70 days in vitro, immediately following induction of oligodendrocytes. At day 90, spheroids were treated with hypoxyprobe, a chemical used to visualize hypoxic regions less

than $1 \% \mathrm{O}_{2}$ (Pogue et al., 2001), and harvested for analysis (Figure 5H). Immunohistochemistry for oligodendrocytes (MYRF+ cells) and hypoxic regions (defined by hypoxyprobe + staining) demonstrated a significant 3.8-fold reduction in the number of MYRF+ oligodendrocytes in hypoxic regions compared to normoxic regions within the spheroids (Figures $5 \mathrm{I}$ and $5 \mathrm{~J}$ ). 
of oligodendrocytes within hypoxic regions of the spheroid (Figures $5 \mathrm{I}$ and $5 \mathrm{~J}$ ). Collectively these results show that oxygen tensions shape oligodendrocyte development and that MEK inhibition circumvents the hypoxia-mediated inhibition of oligodendrocyte formation in 3D models of human brain development.

\section{DISCUSSION}

Cells are equipped to translate external cues from the environment into internal signals that ultimately alter transcriptional programs. Molecular oxygen is crucial to support energy production of the cell, and low oxygen upregulates a rapid and conserved transcriptional response mediated largely by HIF transcription factors in all mammalian cells. HIF1a promotes an adaptive response by upregulating oxygen-independent metabolism and increasing blood vessel formation; however, chronic accumulation of HIF1a negatively impacts the function of almost every organ system (Kullmann et al., 2020; Lee et al., 2019; Menendez-Montes et al., 2016; Takubo et al., 2010).

Here, we profiled the genome-wide functional targets of HIF1a in OPCs and found that HIF1a not only binds to canonical hypoxia response genes that are shared across multiple cell types, but also activates a unique set of non-canonical genes in a cell-type-specific manner. In the context of the brain, these non-canonical HIF1a targets that impair oligodendrocyte formation could have implications in the numerous hypoxia driven pathologies of white matter such as diffuse white matter injury of prematurity (Salmaso et al., 2014; van Tilborg et al., 2018), white matter stroke in adults (Hankey, 2017; Marin and Carmichael, 2018) and multiple sclerosis (Graumann et al., 2003; Zeis et al., 2008). More broadly, we suggest that non-canonical HIF1a targets in diverse cell types impact a variety of cell-type-specific functions, such as oligodendrocyte differentiation, heart morphogenesis and T-cell activation. This concept has previously been overlooked as many studies have focused on canonical targets of HIF1a or those 
that were discovered in immortalized cell lines, which may behave differently in response to HIF1a accumulation.

The same transcription factor can bind to different gene targets in different cell types through interaction with transcriptional machinery unique to each cell type (Mullen et al., 2011; Trompouki et al., 2011). HIF1a binding has been shown to depend on the openness and activation status of chromatin; however, we and others show that chromatin accessibility is not the sole predictor of HIF1a binding (Schodel et al., 2011; Smythies et al., 2019; Xia and Kung, 2009). Our results highlight that HIF1a binds more strongly at core hypoxia response peaks compared to celltype-specific peaks, which are enriched for open chromatin as well as motifs for lineage-defining transcription factors. This implies that acute HIF1a binds more readily to protective pathways in the immediate response to low oxygen, while chronic HIF1a interacts with lineage defining transcription factors to upregulate targets that ultimately impair development and function in a cell-type-specific manner. These non-canonical targets could represent either a pathological "offtarget" effect of sustained HIF1a accumulation or a normal cell-type-specific response to molecular oxygen levels that is coopted in the context of hypoxia disease.

Overall, this work advances our conceptual understanding of the tissue specific response to chronic HIF accumulation and how oxygen tensions regulate tissue physiology and pathology.

\section{ACKNOWLEDGEMENTS}

This research was supported by grants from the National Institutes of Health F30HD096784 (K.C.A), T32NS077888 (K.C.A.), and T32GM007250 (K.C.A) and the New York Stem Cell Foundation (P.J.T. and M.A.S.) as well as institutional support from CWRU School of Medicine and philanthropic support from the Enrile, Peterson, Fakhouri, Long, Goodman, Geller, and Weidenthal families. Additional support was provided by the Small Molecule Drug Development and Genomics core facilities of the CWRU Comprehensive Cancer Center (P30CA043703), the CWRU Light Microscopy Imaging Center (S10-OD016164), and the University of Chicago Genomics Facility. The authors thank Brian Popko for tissue samples (obtained with funding from $\mathrm{NIH}$ grant R01NS034939). We are also grateful to C. Baecher-Allan, J. LaManna, K. Xu, D. Adams, Y. Federov, L. Barbar, E. Prendergast, E. Schwarz, P. Scacheri, D. Neu, E. Cohn, A. Saiakhova, Z. Nevin, M. Elitt, and R. Sallari for technical assistance and/or discussion. 


\section{AUTHOR CONTRIBUTIONS}

K.C.A. and P.J.T. conceived and managed the overall study. K.C.A. and L.R.H. performed, quantified and analyzed all in vitro experiments using mouse OPCs including qPCR, western blot, immunocytochemistry, and generation of CRISPR knockout and CRISPRA OPCs. K.C.A. performed the small molecule screen and dose curve validations. C.F.B. trained K.C.A. to perform ChIP-seq and K.C.A. performed all ChIP-seq experiments in the paper with analysis of data performed by A.R.M and S.H. A.R.M. and M.A.S. assisted with RNA-seq data analysis. I.R.B. performed mass spectroscopy and quantified the data. B.L.C. generated immunopanned in vivo derived OPCs and brain tissue samples. M.M. and A.G. performed oligocortical spheroid experiments. M.A.S. and B.L.C contributed key ideas for experimental design and assembly of figures. K.C.A. assembled all figures and performed statistical analyses. K.C.A. and P.J.T. wrote the manuscript with input from all authors.

\section{DECLARATION OF INTERESTS}

P.J.T. is a co-founder and consultant for Convelo therapeutics, which has licensed unrelated patents from Case Western Reserve University. P.J.T and Case Western Reserve University hold equity in Convelo Therapeutics. All other authors have no competing interests.

\section{REFERENCES}

Baas, D., Bourbeau, D., Sarlieve, L.L., Ittel, M.E., Dussault, J.H., and Puymirat, J. (1997).

Oligodendrocyte maturation and progenitor cell proliferation are independently regulated by thyroid hormone. Glia 19, 324-332.

Barres, B.A., Hart, I.K., Coles, H.S., Burne, J.F., Voyvodic, J.T., Richardson, W.D., and Raff, M.C. (1992). Cell death and control of cell survival in the oligodendrocyte lineage. Cell 70, 31-46.

Barres, B.A., Lazar, M.A., and Raff, M.C. (1994). A novel role for thyroid hormone, glucocorticoids and retinoic acid in timing oligodendrocyte development. Development 120, 1097-1108.

Bartlett, H., Veenstra, G.J., and Weeks, D.L. (2010). Examining the cardiac NK-2 genes in early heart development. Pediatr Cardiol 31, 335-341.

Brawner, A.T., Xu, R., Liu, D., and Jiang, P. (2017). Generating CNS organoids from human induced pluripotent stem cells for modeling neurological disorders. Int J Physiol Pathophysiol Pharmacol 9, 101111.

Cassavaugh, J., and Lounsbury, K.M. (2011). Hypoxia-mediated biological control. J Cell Biochem 112, 735-744.

Chang, K.J., Redmond, S.A., and Chan, J.R. (2016). Remodeling myelination: implications for mechanisms of neural plasticity. Nat Neurosci 19, 190-197.

Choudhry, H., and Harris, A.L. (2018). Advances in Hypoxia-Inducible Factor Biology. Cell Metab 27, 281298.

Ciofani, M., Madar, A., Galan, C., Sellars, M., Mace, K., Pauli, F., Agarwal, A., Huang, W., Parkhurst, C.N., Muratet, M., et al. (2012). A validated regulatory network for Th17 cell specification. Cell 151, 289303.

Clayton, B.L., Huang, A., Dukala, D., Soliven, B., and Popko, B. (2017a). Neonatal Hypoxia Results in Peripheral Nerve Abnormalities. Am J Pathol 187, 245-251. 
Clayton, B.L., Huang, A., Kunjamma, R.B., Solanki, A., and Popko, B. (2017b). The integrated stress response in hypoxia-induced diffuse white matter injury. J Neurosci.

Creyghton, M.P., Cheng, A.W., Welstead, G.G., Kooistra, T., Carey, B.W., Steine, E.J., Hanna, J., Lodato, M.A., Frampton, G.M., Sharp, P.A., et al. (2010). Histone H3K27ac separates active from poised enhancers and predicts developmental state. Proc Natl Acad Sci U S A 107, 21931-21936.

Dengler, V.L., Galbraith, M., and Espinosa, J.M. (2014). Transcriptional regulation by hypoxia inducible factors. Crit Rev Biochem Mol Biol 49, 1-15.

Doench, J.G., Fusi, N., Sullender, M., Hegde, M., Vaimberg, E.W., Donovan, K.F., Smith, I., Tothova, Z., Wilen, C., Orchard, R., et al. (2016). Optimized sgRNA design to maximize activity and minimize off-target effects of CRISPR-Cas9. Nat Biotechnol 34, 184-191.

Emery, B. (2010). Regulation of oligodendrocyte differentiation and myelination. Science 330, 779-782.

Fancy, S.P., Harrington, E.P., Yuen, T.J., Silbereis, J.C., Zhao, C., Baranzini, S.E., Bruce, C.C., Otero, J.J., Huang, E.J., Nusse, R., et al. (2011). Axin2 as regulatory and therapeutic target in newborn brain injury and remyelination. Nat Neurosci 14, 1009-1016.

Gao, F.B., Apperly, J., and Raff, M. (1998). Cell-intrinsic timers and thyroid hormone regulate the probability of cell-cycle withdrawal and differentiation of oligodendrocyte precursor cells. Dev Biol 197, 5466.

Giandomenico, S.L., and Lancaster, M.A. (2017). Probing human brain evolution and development in organoids. Curr Opin Cell Biol 44, 36-43.

Graumann, U., Reynolds, R., Steck, A.J., and Schaeren-Wiemers, N. (2003). Molecular changes in normal appearing white matter in multiple sclerosis are characteristic of neuroprotective mechanisms against hypoxic insult. Brain Pathol 13, 554-573.

Grinspan, J.B., Edell, E., Carpio, D.F., Beesley, J.S., Lavy, L., Pleasure, D., and Golden, J.A. (2000). Stage-specific effects of bone morphogenetic proteins on the oligodendrocyte lineage. J Neurobiol 43, 1 17.

Guimaraes-Camboa, N., Stowe, J., Aneas, I., Sakabe, N., Cattaneo, P., Henderson, L., Kilberg, M.S., Johnson, R.S., Chen, J., McCulloch, A.D., et al. (2015). HIF1alpha Represses Cell Stress Pathways to Allow Proliferation of Hypoxic Fetal Cardiomyocytes. Dev Cell 33, 507-521.

Haase, V.H. (2009). The VHL tumor suppressor: master regulator of HIF. Curr Pharm Des 15, 3895-3903.

Hankey, G.J. (2017). Stroke. Lancet 389, 641-654.

Heinz, S., Benner, C., Spann, N., Bertolino, E., Lin, Y.C., Laslo, P., Cheng, J.X., Murre, C., Singh, H., and Glass, C.K. (2010). Simple combinations of lineage-determining transcription factors prime cis-regulatory elements required for macrophage and B cell identities. Mol Cell 38, 576-589.

Horlbeck, M.A., Gilbert, L.A., Villalta, J.E., Adamson, B., Pak, R.A., Chen, Y., Fields, A.P., Park, C.Y., Corn, J.E., Kampmann, M., et al. (2016). Compact and highly active next-generation libraries for CRISPRmediated gene repression and activation. Elife 5.

Huang, L.E. (2008). Carrot and stick: HIF-alpha engages c-Myc in hypoxic adaptation. Cell Death Differ $15,672-677$. 
Hubler, Z., Allimuthu, D., Bederman, I., Elitt, M.S., Madhavan, M., Allan, K.C., Shick, H.E., Garrison, E., M, T.K., Factor, D.C., et al. (2018). Accumulation of 8,9-unsaturated sterols drives oligodendrocyte formation and remyelination. Nature 560, 372-376.

Ivan, M., Kondo, K., Yang, H., Kim, W., Valiando, J., Ohh, M., Salic, A., Asara, J.M., Lane, W.S., and Kaelin, W.G., Jr. (2001). HIFalpha targeted for VHL-mediated destruction by proline hydroxylation: implications for O2 sensing. Science 292, 464-468.

Jaakkola, P., Mole, D.R., Tian, Y.M., Wilson, M.I., Gielbert, J., Gaskell, S.J., von Kriegsheim, A., Hebestreit, H.F., Mukherji, M., Schofield, C.J., et al. (2001). Targeting of HIF-alpha to the von HippelLindau ubiquitylation complex by O2-regulated prolyl hydroxylation. Science 292, 468-472.

Jablonska, B., Gierdalski, M., Chew, L.J., Hawley, T., Catron, M., Lichauco, A., Cabrera-Luque, J., Yuen, T., Rowitch, D., and Gallo, V. (2016). Sirt1 regulates glial progenitor proliferation and regeneration in white matter after neonatal brain injury. Nat Commun 7, 13866.

Jablonska, B., Scafidi, J., Aguirre, A., Vaccarino, F., Nguyen, V., Borok, E., Horvath, T.L., Rowitch, D.H., and Gallo, V. (2012). Oligodendrocyte regeneration after neonatal hypoxia requires FoxO1-mediated p27Kip1 expression. J Neurosci 32, 14775-14793.

Joung, J., Konermann, S., Gootenberg, J.S., Abudayyeh, O.O., Platt, R.J., Brigham, M.D., Sanjana, N.E., and Zhang, F. (2017). Genome-scale CRISPR-Cas9 knockout and transcriptional activation screening. Nat Protoc 12, 828-863.

Kaelin, W.G., Jr., and Ratcliffe, P.J. (2008). Oxygen sensing by metazoans: the central role of the HIF hydroxylase pathway. Mol Cell 30, 393-402.

Kaluz, S., Kaluzova, M., and Stanbridge, E.J. (2003). Expression of the hypoxia marker carbonic anhydrase IX is critically dependent on SP1 activity. Identification of a novel type of hypoxia-responsive enhancer. Cancer Res 63, 917-922.

Kullmann, J.A., Trivedi, N., Howell, D., Laumonnerie, C., Nguyen, V., Banerjee, S.S., Stabley, D.R., Shirinifard, A., Rowitch, D.H., and Solecki, D.J. (2020). Oxygen Tension and the VHL-Hif1alpha Pathway Determine Onset of Neuronal Polarization and Cerebellar Germinal Zone Exit. Neuron.

Kupferschmidt, K. (2019). Cellular oxygen sensor system earns Nobel for trio. Science 366, 167.

Lager, A.M., Corradin, O.G., Cregg, J.M., Elitt, M.S., Shick, H.E., Clayton, B.L.L., Allan, K.C., Olsen, H.E., Madhavan, M., and Tesar, P.J. (2018). Rapid functional genetics of the oligodendrocyte lineage using pluripotent stem cells. Nat Commun 9, 3708.

Lee, J.W., Ko, J., Ju, C., and Eltzschig, H.K. (2019). Hypoxia signaling in human diseases and therapeutic targets. Exp Mol Med 51, 68.

Lee, Y.S., Kim, J.W., Osborne, O., Oh, D.Y., Sasik, R., Schenk, S., Chen, A., Chung, H., Murphy, A., Watkins, S.M., et al. (2014). Increased adipocyte O2 consumption triggers HIF-1alpha, causing inflammation and insulin resistance in obesity. Cell 157, 1339-1352.

Levy, C., Khaled, M., and Fisher, D.E. (2006). MITF: master regulator of melanocyte development and melanoma oncogene. Trends Mol Med 12, 406-414.

Liddelow, S.A., Guttenplan, K.A., Clarke, L.E., Bennett, F.C., Bohlen, C.J., Schirmer, L., Bennett, M.L., Munch, A.E., Chung, W.S., Peterson, T.C., et al. (2017). Neurotoxic reactive astrocytes are induced by activated microglia. Nature 541, 481-487. 
Loftus, S.K., Baxter, L.L., Cronin, J.C., Fufa, T.D., Program, N.C.S., and Pavan, W.J. (2017). Hypoxiainduced HIF1alpha targets in melanocytes reveal a molecular profile associated with poor melanoma prognosis. Pigment Cell Melanoma Res 30, 339-352.

Madhavan, M., Nevin, Z.S., Shick, H.E., Garrison, E., Clarkson-Paredes, C., Karl, M., Clayton, B.L.L., Factor, D.C., Allan, K.C., Barbar, L., et al. (2018). Induction of myelinating oligodendrocytes in human cortical spheroids. Nature methods 15, 700-706.

Majmundar, A.J., Wong, W.J., and Simon, M.C. (2010). Hypoxia-inducible factors and the response to hypoxic stress. Mol Cell 40, 294-309.

Marin, M.A., and Carmichael, S.T. (2018). Mechanisms of demyelination and remyelination in the young and aged brain following white matter stroke. Neurobiol Dis.

Mei, F., Fancy, S.P.J., Shen, Y.A., Niu, J., Zhao, C., Presley, B., Miao, E., Lee, S., Mayoral, S.R., Redmond, S.A., et al. (2014). Micropillar arrays as a high-throughput screening platform for therapeutics in multiple sclerosis. Nat Med 20, 954-960.

Menendez-Montes, I., Escobar, B., Palacios, B., Gomez, M.J., Izquierdo-Garcia, J.L., Flores, L., JimenezBorreguero, L.J., Aragones, J., Ruiz-Cabello, J., Torres, M., et al. (2016). Myocardial VHL-HIF Signaling Controls an Embryonic Metabolic Switch Essential for Cardiac Maturation. Dev Cell 39, 724-739.

Miska, J., Lee-Chang, C., Rashidi, A., Muroski, M.E., Chang, A.L., Lopez-Rosas, A., Zhang, P., Panek, W.K., Cordero, A., Han, Y., et al. (2019). HIF-1alpha Is a Metabolic Switch between Glycolytic-Driven Migration and Oxidative Phosphorylation-Driven Immunosuppression of Tregs in Glioblastoma. Cell Rep 27, 226-237 e224.

Mullen, A.C., Orlando, D.A., Newman, J.J., Loven, J., Kumar, R.M., Bilodeau, S., Reddy, J., Guenther, M.G., DeKoter, R.P., and Young, R.A. (2011). Master transcription factors determine cell-type-specific responses to TGF-beta signaling. Cell 147, 565-576.

Nagao, A., Kobayashi, M., Koyasu, S., Chow, C.C.T., and Harada, H. (2019). HIF-1-Dependent Reprogramming of Glucose Metabolic Pathway of Cancer Cells and Its Therapeutic Significance. Int J Mol Sci 20.

Najm, F.J., Madhavan, M., Zaremba, A., Shick, E., Karl, R.T., Factor, D.C., Miller, T.E., Nevin, Z.S., Kantor, C., Sargent, A., et al. (2015). Drug-based modulation of endogenous stem cells promotes functional remyelination in vivo. Nature 522, 216-220.

Najm, F.J., Zaremba, A., Caprariello, A.V., Nayak, S., Freundt, E.C., Scacheri, P.C., Miller, R.H., and Tesar, P.J. (2011). Rapid and robust generation of functional oligodendrocyte progenitor cells from epiblast stem cells. Nature methods 8, 957-962.

Nave, K.A. (2010). Myelination and the trophic support of long axons. Nat Rev Neurosci 11, 275-283.

Pogue, B.W., Paulsen, K.D., O'Hara, J.A., Wilmot, C.M., and Swartz, H.M. (2001). Estimation of oxygen distribution in RIF-1 tumors by diffusion model-based interpretation of pimonidazole hypoxia and eppendorf measurements. Radiat Res 155, 15-25.

Quinlan, A.R., and Hall, I.M. (2010). BEDTools: a flexible suite of utilities for comparing genomic features. Bioinformatics 26, 841-842.

Ramirez, F., Ryan, D.P., Gruning, B., Bhardwaj, V., Kilpert, F., Richter, A.S., Heyne, S., Dundar, F., and Manke, T. (2016). deepTools2: a next generation web server for deep-sequencing data analysis. Nucleic Acids Res 44, W160-165. 
Rechsteiner, M.P., von Teichman, A., Nowicka, A., Sulser, T., Schraml, P., and Moch, H. (2011). VHL gene mutations and their effects on hypoxia inducible factor HIFalpha: identification of potential driver and passenger mutations. Cancer Res 71, 5500-5511.

Salmaso, N., Jablonska, B., Scafidi, J., Vaccarino, F.M., and Gallo, V. (2014). Neurobiology of premature brain injury. Nat Neurosci 17, 341-346.

Sanjana, N.E., Shalem, O., and Zhang, F. (2014). Improved vectors and genome-wide libraries for CRISPR screening. Nature methods 11, 783-784.

Scafidi, J., Hammond, T.R., Scafidi, S., Ritter, J., Jablonska, B., Roncal, M., Szigeti-Buck, K., Coman, D., Huang, Y., McCarter, R.J., Jr., et al. (2014). Intranasal epidermal growth factor treatment rescues neonatal brain injury. Nature 506, 230-234.

Schmid-Burgk, J.L., Schmidt, T., Gaidt, M.M., Pelka, K., Latz, E., Ebert, T.S., and Hornung, V. (2014). OutKnocker: a web tool for rapid and simple genotyping of designer nuclease edited cell lines. Genome Res 24, 1719-1723.

Schmidt, D., Wilson, M.D., Spyrou, C., Brown, G.D., Hadfield, J., and Odom, D.T. (2009). ChIP-seq: using high-throughput sequencing to discover protein-DNA interactions. Methods 48, 240-248.

Schodel, J., Oikonomopoulos, S., Ragoussis, J., Pugh, C.W., Ratcliffe, P.J., and Mole, D.R. (2011). Highresolution genome-wide mapping of HIF-binding sites by ChIP-seq. Blood 117, e207-217.

Semenza, G.L. (2007). Life with oxygen. Science 318, 62-64.

Semenza, G.L. (2012). Hypoxia-inducible factors in physiology and medicine. Cell 148, 399-408.

Shaul, Y.D., and Seger, R. (2007). The MEK/ERK cascade: from signaling specificity to diverse functions. Biochim Biophys Acta 1773, 1213-1226.

Shindo, A., Liang, A.C., Maki, T., Miyamoto, N., Tomimoto, H., Lo, E.H., and Arai, K. (2016). Subcortical ischemic vascular disease: Roles of oligodendrocyte function in experimental models of subcortical whitematter injury. J Cereb Blood Flow Metab 36, 187-198.

Smythies, J.A., Sun, M., Masson, N., Salama, R., Simpson, P.D., Murray, E., Neumann, V., Cockman, M.E., Choudhry, H., Ratcliffe, P.J., et al. (2019). Inherent DNA-binding specificities of the HIF-1alpha and HIF-2alpha transcription factors in chromatin. EMBO Rep 20.

Stolt, C.C., Lommes, P., Friedrich, R.P., and Wegner, M. (2004). Transcription factors Sox8 and Sox10 perform non-equivalent roles during oligodendrocyte development despite functional redundancy. Development 131, 2349-2358.

Stolt, C.C., Rehberg, S., Ader, M., Lommes, P., Riethmacher, D., Schachner, M., Bartsch, U., and Wegner, M. (2002). Terminal differentiation of myelin-forming oligodendrocytes depends on the transcription factor Sox10. Genes Dev 16, 165-170.

Tabula Muris, C., Overall, c., Logistical, c., Organ, c., processing, Library, p., sequencing, Computational data, a., Cell type, a., Writing, g., et al. (2018). Single-cell transcriptomics of 20 mouse organs creates a Tabula Muris. Nature 562, 367-372.

Takubo, K., Goda, N., Yamada, W., Iriuchishima, H., Ikeda, E., Kubota, Y., Shima, H., Johnson, R.S., Hirao, A., Suematsu, M., et al. (2010). Regulation of the HIF-1alpha level is essential for hematopoietic stem cells. Cell Stem Cell 7, 391-402. 
Trompouki, E., Bowman, T.V., Lawton, L.N., Fan, Z.P., Wu, D.C., DiBiase, A., Martin, C.S., Cech, J.N., Sessa, A.K., Leblanc, J.L., et al. (2011). Lineage regulators direct BMP and Wnt pathways to cell-specific programs during differentiation and regeneration. Cell 147, 577-589.

van Tilborg, E., de Theije, C.G.M., van Hal, M., Wagenaar, N., de Vries, L.S., Benders, M.J., Rowitch, D.H., and Nijboer, C.H. (2018). Origin and dynamics of oligodendrocytes in the developing brain: Implications for perinatal white matter injury. Glia 66, 221-238.

Volpe, J.J. (2009). Brain injury in premature infants: a complex amalgam of destructive and developmental disturbances. Lancet Neurol 8, 110-124.

Volpe, J.J., Kinney, H.C., Jensen, F.E., and Rosenberg, P.A. (2011). The developing oligodendrocyte: key cellular target in brain injury in the premature infant. Int J Dev Neurosci 29, 423-440.

Wu, Y., Tang, D., Liu, N., Xiong, W., Huang, H., Li, Y., Ma, Z., Zhao, H., Chen, P., Qi, X., et al. (2017). Reciprocal Regulation between the Circadian Clock and Hypoxia Signaling at the Genome Level in Mammals. Cell Metab 25, 73-85.

Xia, X., and Kung, A.L. (2009). Preferential binding of HIF-1 to transcriptionally active loci determines celltype specific response to hypoxia. Genome Biol 10, R113.

Yohe, M.E., Gryder, B.E., Shern, J.F., Song, Y.K., Chou, H.C., Sindiri, S., Mendoza, A., Patidar, R., Zhang, X., Guha, R., et al. (2018). MEK inhibition induces MYOG and remodels super-enhancers in RASdriven rhabdomyosarcoma. Sci Transl Med 10.

Yu, Y., Chen, Y., Kim, B., Wang, H., Zhao, C., He, X., Liu, L., Liu, W., Wu, L.M., Mao, M., et al. (2013). Olig2 targets chromatin remodelers to enhancers to initiate oligodendrocyte differentiation. Cell 152, 248261.

Yuen, T.J., Silbereis, J.C., Griveau, A., Chang, S.M., Daneman, R., Fancy, S.P., Zahed, H., Maltepe, E., and Rowitch, D.H. (2014). Oligodendrocyte-encoded HIF function couples postnatal myelination and white matter angiogenesis. Cell 158, 383-396.

Zeis, T., Graumann, U., Reynolds, R., and Schaeren-Wiemers, N. (2008). Normal-appearing white matter in multiple sclerosis is in a subtle balance between inflammation and neuroprotection. Brain 131, 288303.

Zhang, S., Kim, B., Zhu, X., Gui, X., Wang, Y., Lan, Z., Prabhu, P., Fond, K., Wang, A., and Guo, F. (2020). Glial type specific regulation of CNS angiogenesis by HIFa-activated different signaling pathways. bioRxiv, 2020.2001.2031.929661.

Zhang, Y., Chen, K., Sloan, S.A., Bennett, M.L., Scholze, A.R., O'Keeffe, S., Phatnani, H.P., Guarnieri, P., Caneda, C., Ruderisch, N., et al. (2014). An RNA-sequencing transcriptome and splicing database of glia, neurons, and vascular cells of the cerebral cortex. J Neurosci 34, 11929-11947. 


\section{METHODS}

584

\section{OPC preparation and culture}

OPCs were generated from the epiblast stem cells (EpiSCs) as previously described (Najm et al., 2011). These EpiSC derived OPCs were sorted to purity by fluorescence activated cell sorting using conjugated CD140a-APC (eBioscience, 17-1401; 1:80) and NG2-AF488 (Millipore, AB5320A4; $1: 100$ ) antibodies. Primary mouse OPCs were derived using two methods. In the first method, cerebral cortices were harvested from postnatal day 2 (P2) C57BL/6J pups and dissociated using a Tumor Dissociation Kit (Miltenyi). Cells were then filtered through a $70 \mu \mathrm{m}$ filter, washed in DMEM/F12, and plated on poly-ornithine (PO) (Sigma, P3655-50MG) and laminin (Sigma, L2020-1MG) coated plates to be expanded, passaged, and used in experiments. The second method follows the immunopanning protocol from P7 C57BL/6J mice (Barres et al., 1992). Primary in vivo derived OPCs using either method were pooled from multiple pups such that they are a combination of male and female cells.

All OPCs were grown on PO and laminin coated flasks in growth media consisting of DMEM/F12 supplemented with N2 Max (R\&D Systems), B27 (Thermo Fisher), 20ng/mL bFGF (R\&D Systems), and 20ng/mL PDGFA (R\&D Systems). Media was changed every 48 hours.

\section{Mouse OPC differentiation to oligodendrocytes and astrocytes}

For oligodendrocyte generation, OPCs were seeded at either 40,000 cells per well (96-well plate, Fisher, 167008) or 15,000 cells per well (384-well PDL-coated cell carrier plates, PerkinElmer, 6057500) on plates coated with PO and laminin. Oligodendrocyte differentiation media consisted of DMEM/F12 supplemented with N2 Max, B27, 100ng/mL noggin (R\&D, 3344NG050), 100ng/mL IGF-1 (R\&D, 291G1200), 10uM cyclic AMP (Sigma, D0260-100MG), 10ng/mL NT3 (R\&D, 267N3025) and 40ng/mL T3 (Thyroid hormone, Sigma, T-6397). Cells were analyzed after 3 days unless otherwise noted. 

supplemented with sodium pyruvate, glutamax, N2 Max, and N-acetyl-cysteine and with growth factors including 20ng/mL bFGF, 5ng/mL Hb-EGF (R\&D, 259-HE-050), 10ng/mL CNTF (R\&D, 557-NT-010), and 10ng/mL BMP4 (R\&D, 314-BP-050) for 3 days.

\section{In vitro hypoxia experiments}

OPCs were plated in OPC growth media and then placed into a 2 shelf $\mathrm{C}$-Chamber from BioSpherix (C-274). Oxygen tension was controlled using the ProOx 110 from BioSpherix such that nitrogen gas would flush out oxygen to maintain the chamber at the desired oxygen level. The subchamber was set at $1 \% \mathrm{O}_{2}$ and cells were cultured for 48 hours unless otherwise noted. Hypoxia treated cells were then rapidly lysed for RNA or protein to minimize degradation of HIFs upon exposure to room air. The normoxic controls were cultured concurrently in the same cell culture incubator containing the BioSpherix C-chamber.

624

\section{Immunocytochemistry}

For antigens requiring live staining (O1 and 04$)$, antibodies were diluted in N2B27 base media supplemented with 10\% Donkey Serum (v/v) (017-000-121, Jackson ImmunoResearch) and then added to cells for 18 minutes at $37^{\circ} \mathrm{C}$. Cells were then fixed in cold $4 \%$ PFA (Electron microscopy sciences) for 18 minutes at room temperature, washed with PBS, and permeabilized and blocked in blocking solution containing $0.1 \%$ Triton X-100 in PBS supplemented with $10 \%$ normal donkey serum (v/v) for 30 minutes at room temperature. Primary antibodies were diluted in blocking solution and incubated with samples overnight at $4^{\circ} \mathrm{C}$. Primary antibodies used included anti-

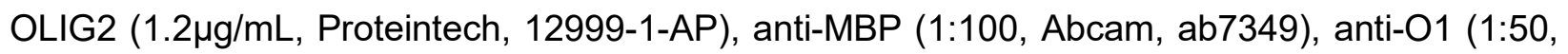
CCF Hybridoma Core), anti-O4 (1:100, CCF Hybridoma Core), anti-ASCL2 (1:10, EMD Millipore, 

and incubated in blocking solution for one hour with the appropriate secondary antibody conjugated to an Alexa-Fluor (4ug/mL, Thermo Fisher) along with the nuclear stain DAPI (Sigma, $1 \mathrm{ug} / \mathrm{mL})$.

\section{High content imaging and quantification}

Both 96-well and 384-well plates were imaged using the Operetta High Content imaging and analysis system (PerkinElmer). For 96-well and 384-well plates, 8 fields and 5-fields were captured from each well at 20x magnification respectively. Images were analyzed with PerkinEImer Harmony and Columbus software as described previously (Hubler et al., 2018; Najm et al., 2015). In brief, nuclei of live cells were identified using a threshold for area of DAPI staining to exclude pyknotic nuclei or debris. To identify oligodendrocytes, each DAPI positive nucleus was expanded by $50 \%$ to determine potential intersection with staining of an oligodendrocyte marker (O4/O1/MBP) in a separate channel. Expanded nuclei that intersected O4/O1/MBP staining were scored as oligodendrocytes. Percentage of oligodendrocytes was then calculated

\section{Generation of CRISPRKOI CRISPRA OPCs}

Guide sequences were curated from the Brie library (Doench et al., 2016) and cloned into the CRISPRv2 backbone (Addgene 52961) (Sanjana et al., 2014) for generating CRISPR-mediated knockout OPCs. Guide sequences were curated from the CRISPRav2 library (Horlbeck et al., 2016) and cloned into the lenti-SAMv2 backbone (Addgene 75112) (Joung et al., 2017) for CRISPR activation. The activation helper plasmid (Addgene 89308) (Joung et al., 2017) was cotransduced for all CRISPR activation OPCs. Plasmids containing cloning sites for the sgRNA were digested and annealed oligomers containing the sgRNA (indicated in the chart below) were then ligated to their respective backbones. Positive bacterial clones were confirmed to have the 
correct inserted oligomer using Sanger sequencing. HEK293T cells were then transfected using lenti-X shots following the manufacturer's protocol (Clonetech). The next day, transfection media was switched to OPC growth media without growth factors for virus collection. After 2 days, the media from transfected HEK293T cells was collected, filtered, and supplemented with OPC growth factors PDGF and FGF. This lentivirus-containing media was added to OPCs at a ratio of $1: 2(\mathrm{v} / \mathrm{v})$ with fresh OPC growth media. After 24 hours of incubation with virus, transduced cells were switched to fresh OPC growth media and allowed to recover for 48 hours. OPCs were then selected for 96 hours in OPC growth media supplemented with a lethal dose of puromycin (500ng/mL, Invitrogen) for CRISPR knockout cells or a lethal dose of blasticidin $(10 \mu \mathrm{g} / \mathrm{mL}$,

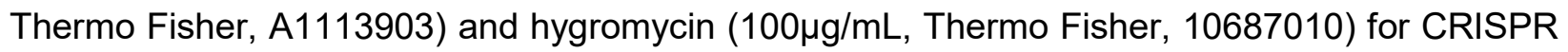
activation cells. OPCs were then allowed to recover for at least 24 hours following removal of selection and frozen down in aliquots for future use. For all experiments, the lentivirally transduced CRISPR/CRISPRA targeting and non-targeting control OPCs were derived from the same batch of OPCs and infected and selected simultaneously. qPCR was performed to validate a reduction generated.

\begin{tabular}{|l|l|}
\hline OPC CRISPR ID & sgRNA sequence (5' to $\mathbf{3}^{\prime}$ ) \\
\hline Cas9 control & empty \\
\hline sgVhl & GCCCGGTGGTAAGATCGGGT \\
\hline sgVhl.2 & TGTCCATCGACATTGAGGGA \\
\hline CRISPRA-NTC & GATCGGTTATGTTTAGGGTT \\
\hline CRISPRA-Ascl2 & CCAAGTTTACCAGCTTTACG \\
\hline CRISPRA-DIx3 & GTTAGGGGTAACAACAAAGA \\
\hline CRISPRA-Vegfa & GAGGCCGTGGACCCTGGTAA \\
\hline CRISPRA-SIc16a3 & GCCTGGCCGCTGTTCCCCGA \\
\hline
\end{tabular}

679 Primers were identified surrounding the target cut site for the two sgVhl constructs (see previous section for sgRNAs) that generate $200-250$ base pair amplicons. 
For the sgVhl.2 cut site the primers were:

PCR products were run on an agarose gel, excised by gel extraction (28115, Qiagen), and submitted to the Case Western Reserve University Genomics core for library preparation and sequencing. Libraries were prepared by adding unique indices by PCR using KAPA HiFi HotStart ReadyMix. Samples were then pooled evenly, quantified using NEBNext@ Library Quant Kit for Illumina ${ }^{\circledR}($ E7630, New England 641 Biolabs), and denatured and diluted per Illumina's MiSeq instructions. These finished libraries were then sequenced using an Illumina MiSeq (250bp paired-end). Results were analyzed using Outknocker software (Schmid-Burgk et al., 2014) (http://www.outknocker.org/outknocker2.htm) to calculate the percentage of reads with insertions or deletions at the sgRNA target site.

\section{Compound screening and assessment}

Compound screening was carried out as described in (Lager et al., 2018). Poly-D-lysine-coated 384-well CellCarrier Ultra plates (PerkinEImer) were coated with laminin diluted in N2B27 base media using an EL406 Microplate Washer Dispenser (BioTek) equipped with a $5 \mu$ lispense cassette (BioTek) and were incubated at $37^{\circ} \mathrm{C}$ for at least 1 hour. A $3 \mathrm{mM}$ stock of the Selleck bioactive library in dimethylsulfoxide (DMSO) was then added to the plates using a 50nL solid pin tool attached to a Janus automated workstation (Perkin Elmer) at a 1:1000 dilution such that each well received a single compound at a final concentration of $3 \mu \mathrm{M}$. Compounds for dose response validation were sourced from the Selleck library, except for ERK1/2 inhibitors SCH772984 
(Selleck, S7101), AZD0364 (Selleck, S8708), and VX-11e (Selleck, S7709) which were purchased separately. OPCs were dispensed in oligodendrocyte differentiation media at 12,500 cells per well into the laminin-coated 384 well plates using the BioTek EL406 Microplate Washer Dispenser and differentiated at $37^{\circ} \mathrm{C}$ for 3 days. At this point, cells were fixed, washed and stained using the BioTek EL406 Microplate Washer Dispenser. Cells were stained with anti-MBP (1:100, Abcam, ab7349) along with DAPI ( $1 \mu \mathrm{g} / \mathrm{ml}$, Sigma) and imaged using the Operetta High Content Imaging and Analysis system (PerkinElmer).

\section{Western blot}

For cell culture derived protein samples, at least 1 million OPCs were collected and lysed in RIPA buffer (Sigma) supplemented with protease and phosphatase inhibitor (78441, Thermo Fisher) for at least 15 minutes and cleared by centrifugation at $13,000 \mathrm{~g}$ at $4^{\circ} \mathrm{C}$ for 15 minutes. Protein concentrations were determined using the Bradford assay (Bio-Rad Laboratories). Protein was then diluted and added to Laemmli loading buffer, boiled at $95^{\circ} \mathrm{C}$ for 5 minutes, run using NuPAGE Bis-Tris gels (NP0335BOX, Thermo Fisher), and then transferred to PVDF membranes (LC2002, Thermo Fisher). Blocking and primary/secondary antibody solutions were performed for at least 30 min with 5\% nonfat drug milk (Nestle Carnation) in TBS plus $0.1 \%$ Tween 20 (TBST). Primary antibodies used included anti-HIF1a (1:500, Abcam, ab2185), anti-phospho-p44/42 (ERK1/2)

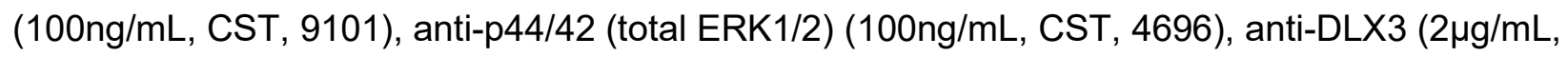
abcam, ab178428), anti-SOX10 (1:100, R\&D, AF2864), anti-B-Actin peroxidase (1:50,000, Sigma, A3854), anti-VHL (5 $\mathrm{gg} / \mathrm{mL}$, BD Biosciences, 556347), anti-ASCL2 (1:10, EMD Millipore, MAB4417), anti-MBP $(1 \mu \mathrm{g} / \mathrm{mL}$, Biolegend, 808401), and anti-MAG $(1 \mu \mathrm{g} / \mathrm{mL}$, Thermo Fisher, 346200). Membranes were then imaged using the LI-COR (Odyssey) and analyzed using Image Studio $^{\mathrm{TM}}$ software that is integrated into the LI-COR imaging suite. Westerns were normalized to loading control Beta-Actin unless otherwise noted. 


\section{qRT-PCR}

At least 500,000 OPCs were lysed in TRIzol (Ambion) followed by phenol-chloroform extraction and processing with the RNeasy Mini Kit (74104, Qiagen). RNA quality and quantity was determined using a NanoDrop spectrophotometer. cDNA was generated using the iSCRIPT kit following the manufacturer's instructions (1708891, Biorad). qRT-PCR was performed using predesigned TaqMan gene expression assays (Thermo Fisher). Probe cat numbers and IDs are included in the chart below. qPCR was performed using the Applied Biosystems 7300 real-time PCR system and probes were normalized to Rp/13a endogenous control.

\begin{tabular}{|l|l|}
\hline Gene ID & Taqman cat. number \\
\hline Sox10 & Mm00569909_m1 \\
\hline Rpl13a & Mm05910660_g1 \\
\hline Vhl & Mm00494137_m1 \\
\hline Ascl2 & Mm01268891_g1 \\
\hline Tpi1 & Mm00833691_g1 \\
\hline Ciart & Mm01255905_g1 \\
\hline P4ha2 & Mm00477940_m1 \\
\hline Pdk1 & Mm00554300_m1 \\
\hline Pfkl & Mm00435587_m1 \\
\hline Slc16a3 & Mm00446102_m1 \\
\hline Dlx3 & Mm00438428_m1 \\
\hline Vefga & Mm00437306_m1 \\
\hline Bnip3 & Mm01275600_g1 \\
\hline Pdgfra & Mm00440701_m1 \\
\hline Plp1 & Mm01297210_m1 \\
\hline Myrf & Mm01194959_m1 \\
\hline
\end{tabular}

At least 1 million OPCs were lysed in TRIzol and RNA was isolated as described for qPCR. Libraries were prepared following protocols from NEBNext Poly(A) mRNA Magnetic Isolation Module (NEB \#E7490L) and NEBNext Ultra RNA Library Prep Kit for Illumina (NEB\# E7530L). In brief, samples were enriched for mRNA using oligo(dT) beads, which were then fragmented randomly and used for cDNA generation and subsequent second-strand synthesis using a custom second-strand synthesis buffer (Illumina), dNTPs, RNase $\mathrm{H}$ and DNA polymerase I. cDNA 
libraries then went through terminal repair, A-base ligation, adapter ligation, size selection and PCR enrichment. Final libraries were pooled evenly and sequenced on the Illumina NovaSeq with paired-end $150 \mathrm{bp}$ reads with a read-depth of at least 20 million reads per sample.

For gene expression analysis, reads were aligned to the mm10 genome build using salmon 0.14 .1 (https://github.com/COMBINE-lab/salmon) to quantify transcript abundance in transcripts per million (TPM) values. Transcripts were summarized as gene-level TPM abundances with tximport. A gene with TPM $>1$ was considered expressed. Differential expression analysis was then performed using DESEQ2 (https://bioconductor.org/packages/release/bioc/html/DESeq2.html). Significant genes were called based on $\mathrm{p}$-adj and fold change values as described in the results section.

\section{Gene ontology analysis and heatmaps}

Metascape (http://metascape.org/) was used to identify significant pathways from RNAseq data. Identification of critical oligodendrocyte genes that were dysregulated in VHL knockout OPCs (see Figure $3 \mathrm{~A}$ ) was performed by fitting RNA-seq (TPM) to the list of genes under the GO term "Oligodendrocyte Development" (GO: 0014003). This spreadsheet of TPM values was used to make a heatmap using the R-package "pheatmap." Increased genes were those greater than 1.25 fold change whereas decreased genes were those less than 0.75 fold change in sgVhl relative to Cas9 control OPCs. In Figure 5D and 5E, RNA-seq data was fit to the genes within "Oligodendrocyte Development" (GO: 0014003) that were downregulated in sgVhl OPCs compared to Cas9 control OPCs (fold change less than 0.75 ).

\section{Gene set enrichment analysis}

Gene set enrichment analysis (GSEA) scores were generated for gene sets in C5.bp datasets using classic scoring, 1000 gene-set permutations, and signal-to-noise metrics. Normalized 
enrichment scores, false discovery rate and FWER o-values were all calculated by GSEA software (https://www.gsea-msigdb.org/gsea/index.jsp).

\section{ChIP-seq and analysis}

Nuclei isolation and chromatin shearing were performed using the Covaris TruChIP protocol following manufacturer's instructions for the "high-cell" format. In brief, 5-20 million cells (H3K27Ac) or 100 million cells (HIF1a) were crosslinked in "Fixing buffer A" supplemented with $1 \%$ fresh formaldehyde for 10 minutes at room temperature with oscillation and quenched for 5 minutes with "Quench buffer E." These cells were then washed with PBS and either snap frozen protocol. The samples were sonicated with the Covaris S2 using the following settings: $5 \%$ Duty factor 4 intensity for four 60-second cycles. Sheared chromatin was cleared and incubated overnight at 4 degrees with primary antibodies that were pre-incubated with protein $G$ magnetic DynaBeads (Thermo Fisher). Primary antibodies used included anti-H3K27Ac (9ug/sample, Abcam, ab4729) and anti-HIF1a (25ug/sample, Abcam, ab2185). These beads were then washed, eluted, reverse cross-linked and treated with RNase A followed by proteinase K. ChIP DNA was purified using Ampure XP beads (Aline Biosciences, C-1003-5) and then used to prepare Illumina sequencing libraries as described previously (Schmidt et al., 2009). Libraries were sequenced on the Illumina HiSeq2500 with single-end 50bp reads with a read-depth of at least 20 million reads per sample.

For peak calling, reads were quality and adapter trimmed using Trim Galore! Version 0.4.1. Trimmed reads were aligned to $\mathrm{mm} 10$ with Bowtie2 version 2.3.2. Duplicate reads (potential artifacts of PCR in library preparation) were removed using Picard MarkDuplicates. Peaks were called with MACS version 2.1.1 to define broad peaks for histone marks (H3K27Ac) and narrow peaks for transcription factors (HIF1a) and normalized to background genomic DNA with matched 
inputs. Thresholding was set at FDR $<0.001$ for calling both H3K27Ac and HIF1a peaks. Peaks were visualized with the Integrative Genomics Viewer (IGV, Broad Institute). Peaks were assigned to the nearest expressed gene (TPM>1 in Cas9 control or sgVhl OPCs) using bedtools available in Galaxy (https://usegalaxy.org).

\section{Diffbind analysis}

H3K27Ac ChIP-seq was performed in duplicate from two independent batches of Cas9 control and sgVhl OPCs. Differential H3K27Ac analysis between sgVhl and Cas9 control OPCs was

"Diffbind software" (https://bioconductor.org/packages/release/bioc/html/DiffBind.html). A false discovery rate of 0.1 was used to call significantly enriched and depleted regions of H3K27Ac.

\section{Motif enrichment analysis}

Motifs were called under significant HIF1a peaks (FDR<0.001) or regions of significantly gained was used with $1000 \mathrm{bp}$ windows with $\mathrm{mm} 10$ as the reference genome.

\section{HIF1a ChIP-seq overlap analysis}

HIF1a ChIP-seq raw data were re-analyzed for: two HIF1a replicates ChIP-seqs and 1 input of E12.5 heart (GSM1500750, GSM1500751, GSM1500749) (Guimaraes-Camboa et al., 2015), two HIF1a replicates ChIP-seqs and 1 input of melanocyte (GSM2305570, GSM2305571, GSM2305572) (Loftus et al., 2017), and two HIF1a ChIP-seqs and 2 inputs of Th17 cells (GSM1004819, GSM1004991, GSM1004820, GSM1004993) (Ciofani et al., 2012). For each of the HIF1a ChIP-seqs, the peaks were called as described above (MACS2, narrow peak, FDR<0.001). Peaks unique to OPCs were identified by finding peaks not found in the union of all the replicates of every other tissue sample using the bedtools (v 2.25.0) (Quinlan and Hall, 2010) 
"intersect" command. Common peaks across tissues were also identified using the bedtools "intersect". RPCG-normed bigwigs were used to create aggregate plots for HIF1a, ATAC-seq, and H3K27ac using the deeptools (version 3.3.1) (Ramirez et al., 2016) "computeMatrix" and "plotHeatmap" commands, centering on the HIF1a peak locations in the sgVhl OPCs. Genes within $5 \mathrm{~Kb}$ of these HIF1a peaks were called as HIF1a target genes. For melanocytes and T-cells, HIF1a target genes were called as only those at the intersection of both of the available replicates. For embryonic heart there were fewer peaks called compared to the other tissues, so HIF1a gene targets were called as the union of the two available replicates. These gene and peak lists were used to generate venn diagrams using the following webtool: (http://bioinformatics.psb.ugent.be/webtools/Venn/). Cell type specific gene targets of HIF1a were called if the gene was only a target of HIF1a in that specific cell type.

\section{Mass spectrometry for lactate and pyruvate}

OPCs were cultured in OPC growth media and after growth media was removed, cells were washed with ice-cold saline ( 3 times) and collected with $1 \mathrm{ml}$ of $80 \%$ ethanol pre-chilled on dry ice. Cells were frozen at $-80^{\circ} \mathrm{C}$ until analyses. This cell extract was then vortexed and sonicated for 30 seconds on, 30 seconds off, alternating for 10 minutes. Next, cells were pelleted by centrifugation at $4^{\circ} \mathrm{C}$ for $10 \mathrm{~min}$ at $14,000 \mathrm{rpm}$. Supernatant was transferred to GC/MS vials and evaporated to dryness under gentle stream of nitrogen. Keto- and aldehyde groups were reduced by addition of $10 \mu \mathrm{l}$ of $1 \mathrm{~N} \mathrm{NaOH}$ plus $15 \mu \mathrm{NaB}^{2} \mathrm{H}_{4}$ (prepared as $10 \mathrm{mg} / \mathrm{ml}$ in $50 \mathrm{mM} \mathrm{NaOH}$ ). After mixing, samples were incubated at room temperature for 1 hour and then acidified by $55 \mu l$ of $1 \mathrm{~N} \mathrm{HCl}$ by dropping the acid slowly. Next, samples were evaporated to dryness. Next, $50 \mu l$ of methanol were added to precipitate boric acid. Internal standard was added (10 $\mu$ of 17:0 FA, 0.1 $\mathrm{mg} / \mathrm{ml}$ ). Samples were evaporated to dryness and reacted with $40 \mu \mathrm{l}$ of pyridine and $60 \mu \mathrm{l}$ of tertbutylbis(dimethylsilyl) trifluoroacetamide with $10 \%$ trimethylchlorosilane (Regisil) TBDMS at $60^{\circ} \mathrm{C}$ for 1 hour. Resulting TBDMS derivatives were injected into GC/MS. Analyses were then carried 

capillary column $(60 \mathrm{~m} \times 0.25 \mathrm{~mm} \times 0.25 \mu \mathrm{m})$ was used in all assays with a helium flow of 1 $\mathrm{ml} / \mathrm{min}$. Samples were analyzed in Selected Ion Monitoring (SIM) mode using electron impact ionization (EI). Ion dwell time was set to $10 \mathrm{msec}$. Lactate and pyruvate were both measured for all samples.

\section{Tissue from mild chronic hypoxia $(\mathrm{MCH})$ mice} Clayton et al., 2017b; Fancy et al., 2011; Scafidi et al., 2014; Yuen et al., 2014) and protein samples were generously provided by Brian Popko. In brief, postnatal day 3 (P3) C57BI6 pups were placed into a BioSpherix chamber maintained at $10+/-0.5 \%$ O2 by displacement of nitrogen the frontal cortex was isolated as this region has been shown to contain subcortical white matter that is susceptible to hypoxia-induced DWMI (Clayton et al., 2017b; Jablonska et al., 2012; Sanjana et al., 2014; Yuen et al., 2014).

866

\section{Oligocortical spheroid generation and immunohistochemistry}

Human embryonic stem cells (line H7, WiCell) were grown in mTesR1 media and oligocortical spheroids were generated as previously described (Madhavan et al., 2018). Spheroids were treated every other day with DMSO or 300nM AZD8330 between days 70 and 74 and harvested on day 90. 2 hours prior to harvesting, spheroids were treated with 200uM Hypoxyprobe-1 (pimonidazole, Hypoxyprobe Inc, Burlington MA). Spheroids were washed in PBS and fixed overnight in ice cold 4\% Paraformaldehyde and then washed in PBS and cryoprotected in a 30\% sucrose solution. Spheroids were frozen in OCT and sectioned at thickness of $15 \mu \mathrm{m}$. Slides were washed in PBS and incubated overnight with anti-MyRF (1:1000, gift from Michael Wegner) and anti-pimonidazole antibodies (1:250, Hypoxyprobe Inc, Burlington MA) followed by labelling 
877 with appropriate Alexa-Fluor labeled secondary antibodies $(2 \mu \mathrm{g} / \mathrm{mL}$, Thermo Fisher). Images

878 were captured using a Hamamatsu Nanozoomer S60 Slide scanner with NDP 2.0 software.

879 Images spanning the edge to the central region of each oligocortical spheroid were used for 880 analysis. MyRF+ oligodendrocytes in Hypoxyprobe+ and Hypoxyprobe- areas within each image $881 \quad$ were quantified using ImageJ software.

\section{Statistics and replicates}

884 GraphPad Prism was used to perform statistical analyses unless otherwise noted. Statistical tests 885 and replicate descriptions are detailed in each figure legend. In brief, black filled-in circles for bar 886 graphs indicate biological replicates whereas open circles represent technical replicates. 887 Statistics were only performed on samples with biological replicates. Data is typically graphed as mean \pm standard deviation (SD) or \pm standard error of the mean (SEM) as detailed in the figure legend. A p-value less than 0.05 was considered significant unless otherwise noted. 

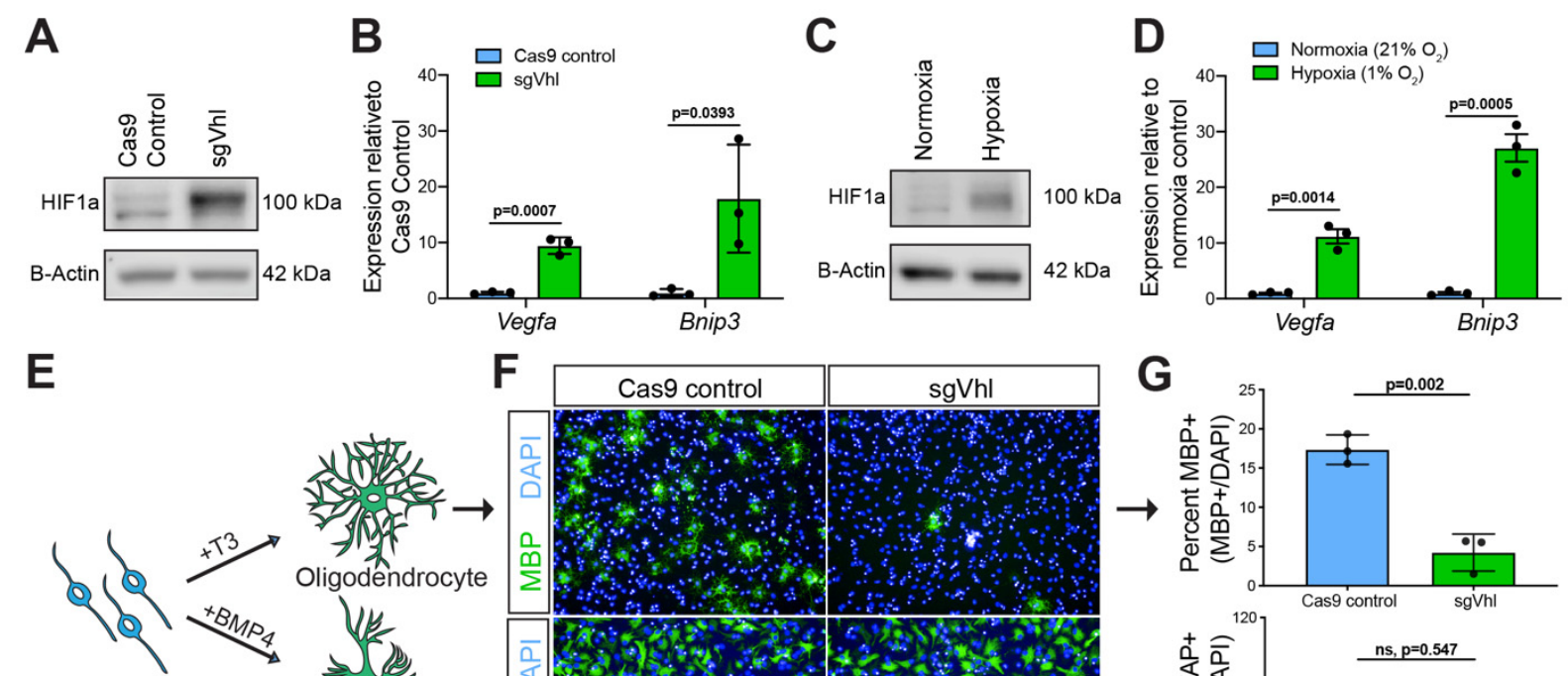

Oligodendrocyte progenitor cells (OPCs)

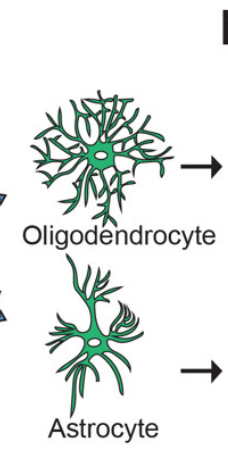

F Cas9 control
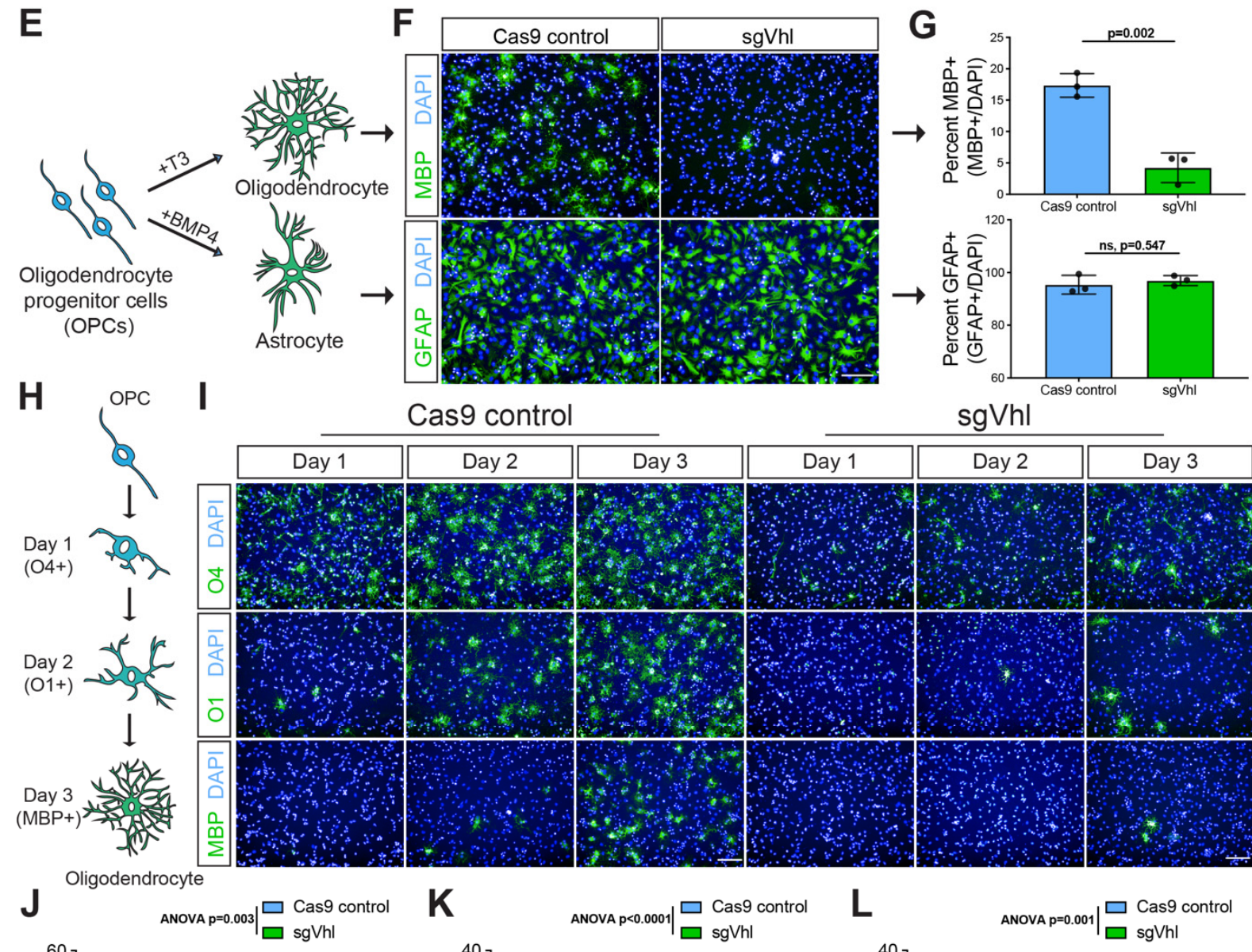

Cas9 control

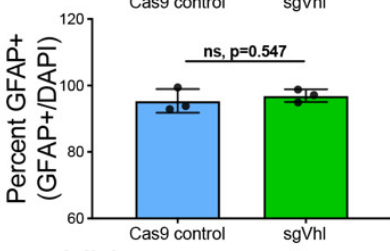
$\mathrm{sgVhl}$
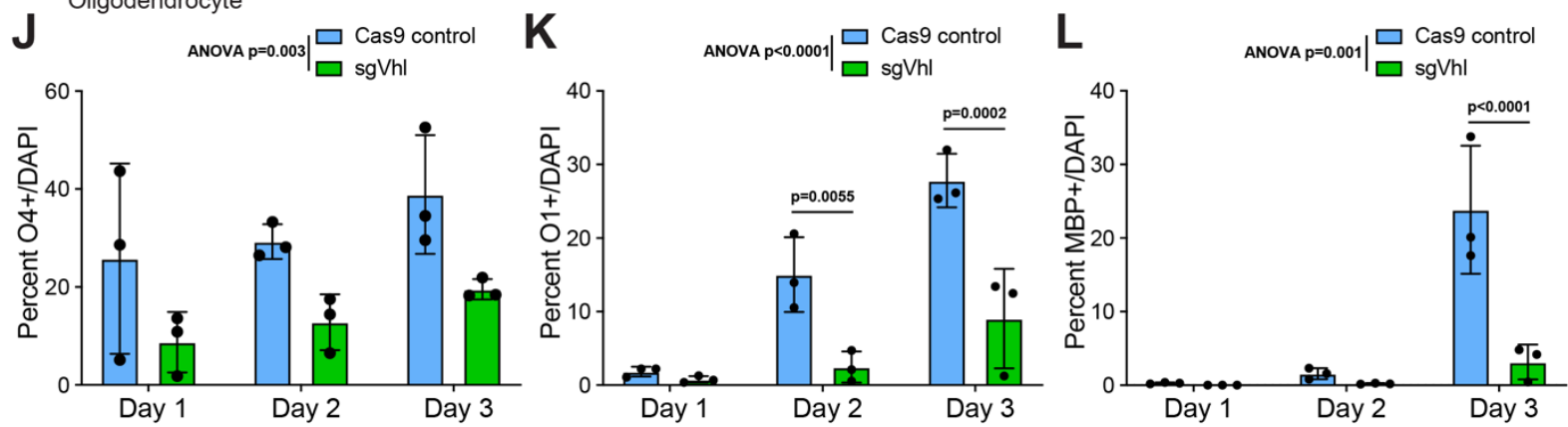
(A) Western blot of HIF1a from nuclear lysates of sgVhl OPCs compared to Cas9 control OPCs with B-Actin as a loading control. Molecular weight is indicated to the right of the blot. 
(B) qRT-PCR of HIF target genes Vegfa and Bnip3 in Cas9 control (in blue) and sgVhl (in green) OPCs normalized to endogenous loading control Rp/13a. Data are presented as mean \pm SEM from 3 independent biological replicates (experiments) with 4 technical replicates (individual wells) per experiment. p-values were calculated using Student's two-tailed t-test.

(C) Western blot of HIF1a from nuclear lysates of OPCs cultured in hypoxia $\left(1 \% \mathrm{O}_{2}\right)$ compared to normoxia $\left(21 \% \mathrm{O}_{2}\right)$ with B-Actin as a loading control. Molecular weight is indicated to the right of the blot.

(D) qRT-PCR of HIF target genes Vegfa and Bnip3 in OPCs cultured in physiological hypoxia (in green) compared to normoxia (in blue) normalized to endogenous loading control Rp/13a. Data are presented as mean \pm SEM from 3 independent biological replicates (experiments) with 4 technical replicates (individual wells) per experiment. p-values were calculated using Student's two-tailed t-test.

(E) Schematic of the two in vitro differentiation schemes directing OPCs to either oligodendrocytes through addition of thyroid hormone (T3) or astrocytes through the addition of BMP4.

(F) Representative images of Cas9 control and sgVhl oligodendrocytes (MBP+ in green) and astrocytes (GFAP+ in green) following 3 days in the respective differentiation media. Nuclei are marked by DAPI (in blue). Scale bar, $100 \mu \mathrm{m}$.

(G) Quantification of the percentage of oligodendrocytes (MBP+ cells / DAPI) and astrocytes (GFAP+ cells / DAPI) formed from sgVhl OPCs (in green) compared to Cas9 control OPCs (in blue). Data are presented as mean \pm SD from 3 independent biological replicates (experiments) with 6-8 technical replicates (individual wells) per experiment. p-values were calculated using Student's two-tailed t-test.

(H) Schematic illustrating acquisition of early (O4), intermediate (O1) and late (MBP) oligodendrocyte markers during the 3-day in vitro oligodendrocyte differentiation procedure in response to addition of T3.

(I) Representative images of early ( $\mathrm{O} 4+$ in green), intermediate $(\mathrm{O} 1+$ in green) and late $(\mathrm{MBP}+$ in green) oligodendrocytes during days 1,2, and 3 of differentiation with Cas9 control and sgVhl

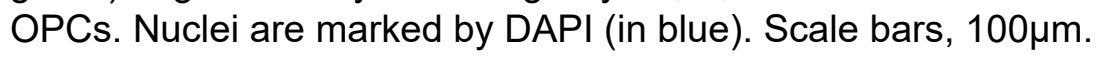

$(\mathrm{J}-\mathrm{L})$ Quantification of the percentage of early $\mathrm{O} 4+(\mathrm{J})$, intermediate $\mathrm{O} 1+(\mathrm{K})$, and late $\mathrm{MBP}+(\mathrm{L})$ oligodendrocytes in sgVhl OPCs (in green) compared to Cas9 control OPCs (in blue) at days 1 , 2 , and 3 of differentiation. Data are presented as mean \pm SD from 3 independent biological replicates (experiments) with 6-8 technical replicates (individual wells) per experiment. To analyze overall differences between Cas9 control and sgVhl OPCs across all timepoints, $p$-values were calculated using two-way ANOVA (reported as ANOVA $p=$ ). To test differences between Cas9 control and sgVhl OPCs at individual timepoints, $p$-values were calculated using Sidak's multiple comparisons test.

See also Figure $\mathbf{S 1 .}$ 

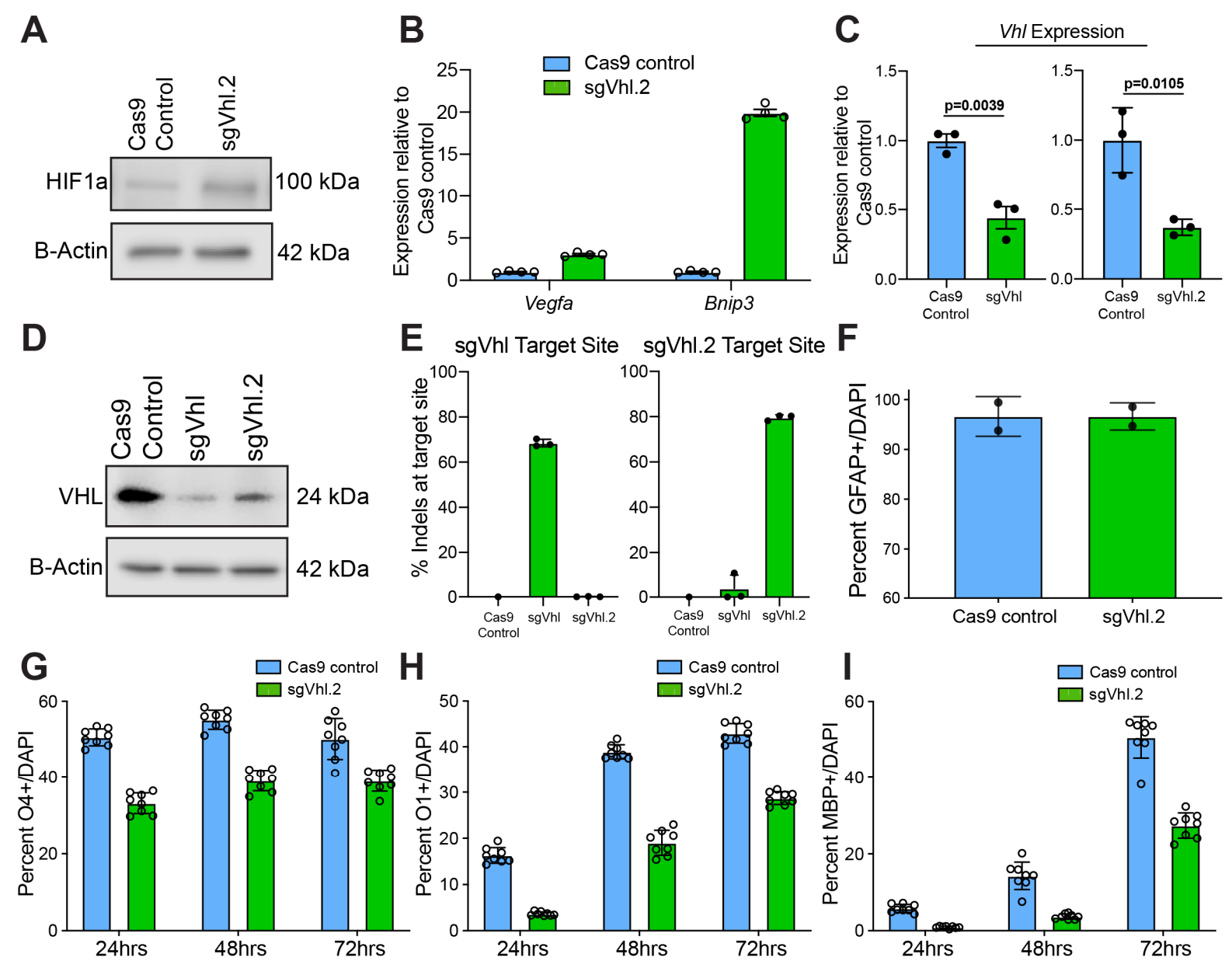

Figure S1. CRISPR Mediated Knockout of Vhl Leads to Loss of VhI Expression and Impairs Oligodendrocyte Formation, Related to Figure 1.

(A) Western blot of HIF1a from nuclear lysates of sgVhl.2 OPCs compared to Cas9 control OPCs with B-Actin as a loading control. Molecular weight is indicated to the right of the blot.

(B) qRT-PCR of HIF target genes Vegfa and Bnip3 in sgVhl.2 OPCs (in green) compared to Cas9 control OPCs (in blue) normalized to endogenous loading control Rp/13a. Data are presented as mean \pm SEM from 4 technical replicates (individual wells).

(C) qRT-PCR of $V h l$ in both sgVhl and sgVhl.2 OPCs (both in green) compared to Cas9 control OPCs (in blue) normalized to endogenous loading control Rp/13a. Data are presented as mean \pm SEM from 3 independent biological replicates (experiments) with 4 technical replicates (individual wells) per experiment. p-values were calculated using Student's two-tailed t-test.

(D) Western blot of VHL from whole cell lysate of sgVhl and sgVhl.2 OPCs compared to Cas9 control OPCs with B-Actin as a loading control. Molecular weight is indicated to the right of the blot. 
(E) In-del analysis utilizing Outknocker software on PCR products surrounding sgRNA cut sites for both sgVhl and sgVhl.2 OPCs compared to Cas9 control. Data are presented as mean \pm SD from 3 independent biological replicates (experiments).

(F) Quantification of the percentage of astrocytes (GFAP+ cells/ DAPI) formed from sgVhl.2 OPCs (in green) compared to Cas9 control OPCs (in blue). Data are presented as mean \pm SD from 2 independent biological replicates (experiments) with 6-8 technical replicates (individual wells) per experiment.

(G-I) Quantification of the percentage of early $\mathrm{O} 4+(\mathrm{G})$, intermediate $\mathrm{O} 1+(\mathrm{H})$, and late MBP+ $(\mathrm{I})$ oligodendrocytes formed from sgVhl.2 OPCs (in green) compared to Cas 9 control OPCs (in blue) at day 1, 2 and 3 of differentiation. Data are presented as mean \pm SD from 8 technical replicates (individual wells) per condition. 
A

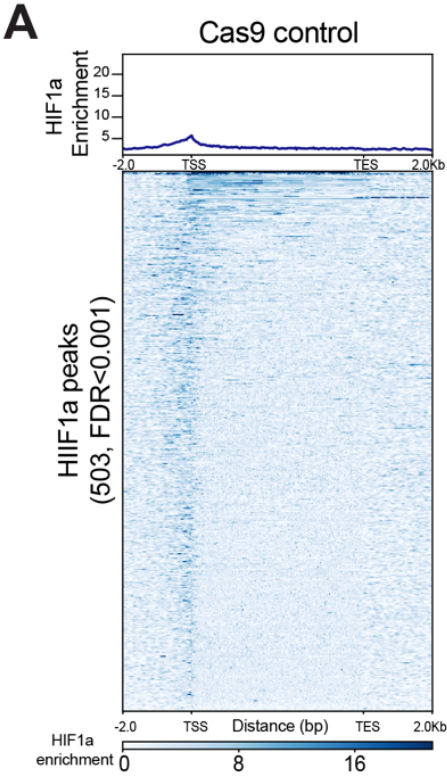

C
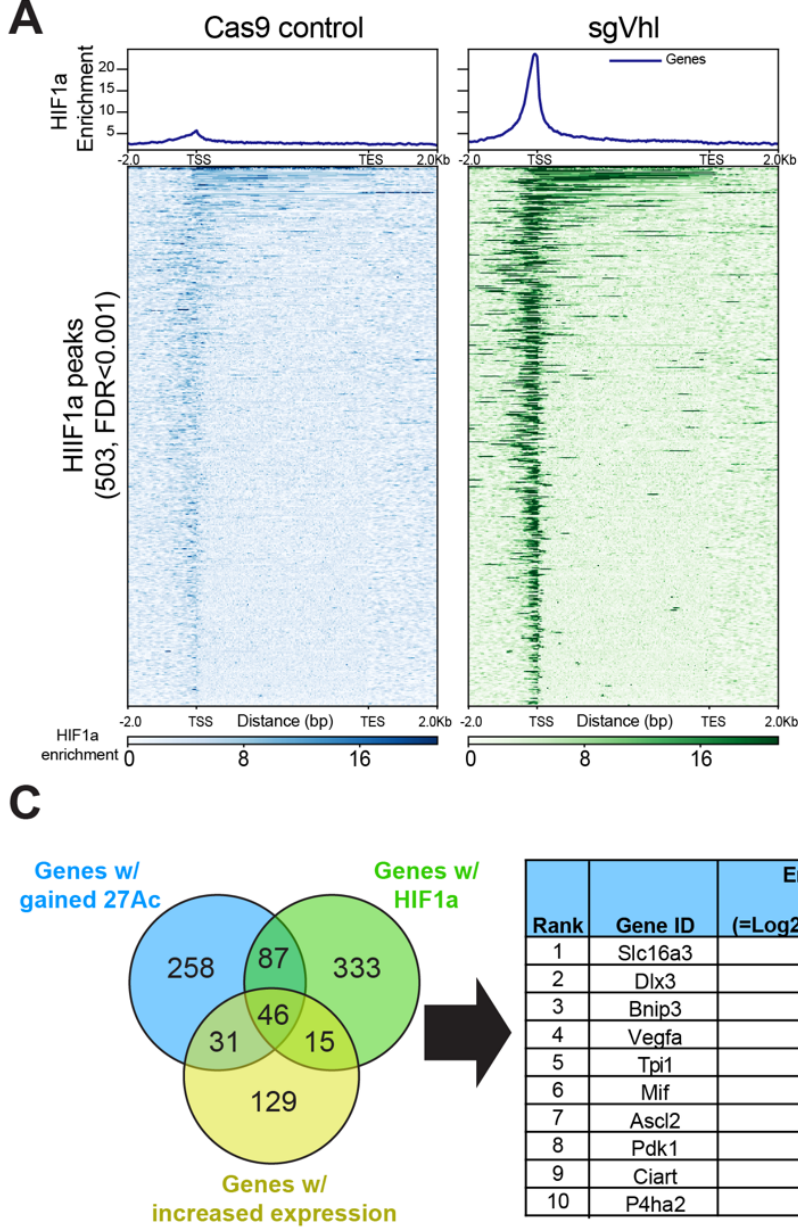

increased expression
B

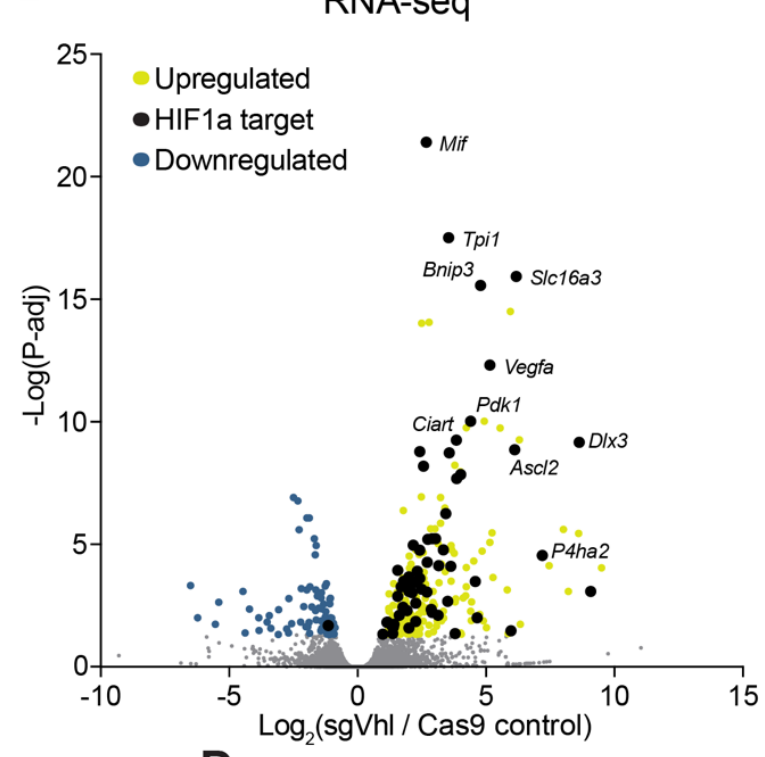

D $\square$ Normoxia $\left(21 \% \mathrm{O}_{2}\right) \square$ Hypoxia $\left(1 \% \mathrm{O}_{2}\right)$

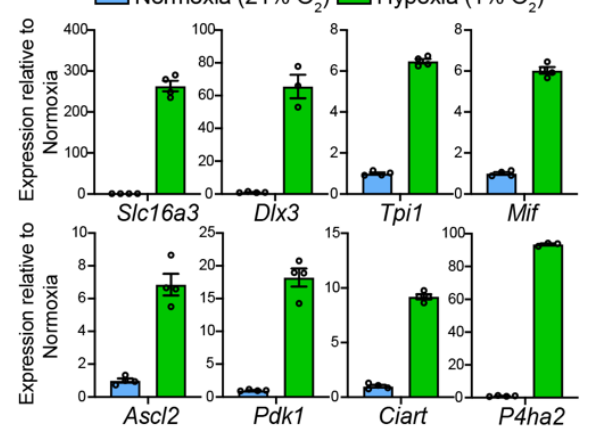

960
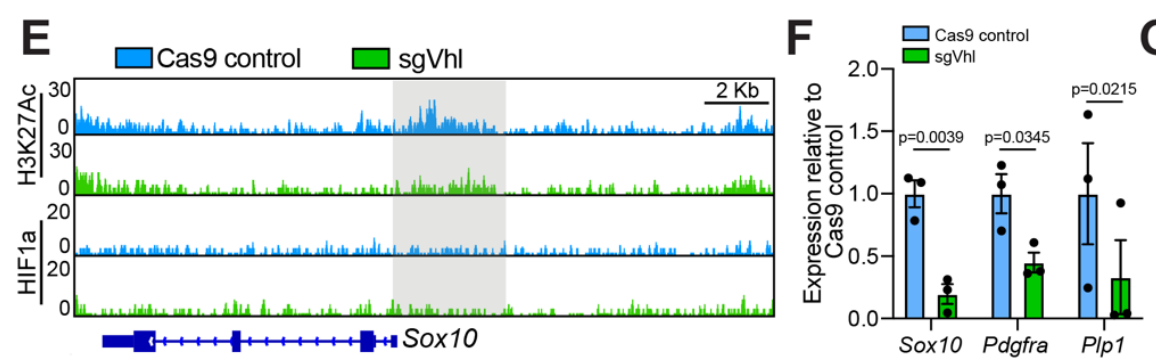

G
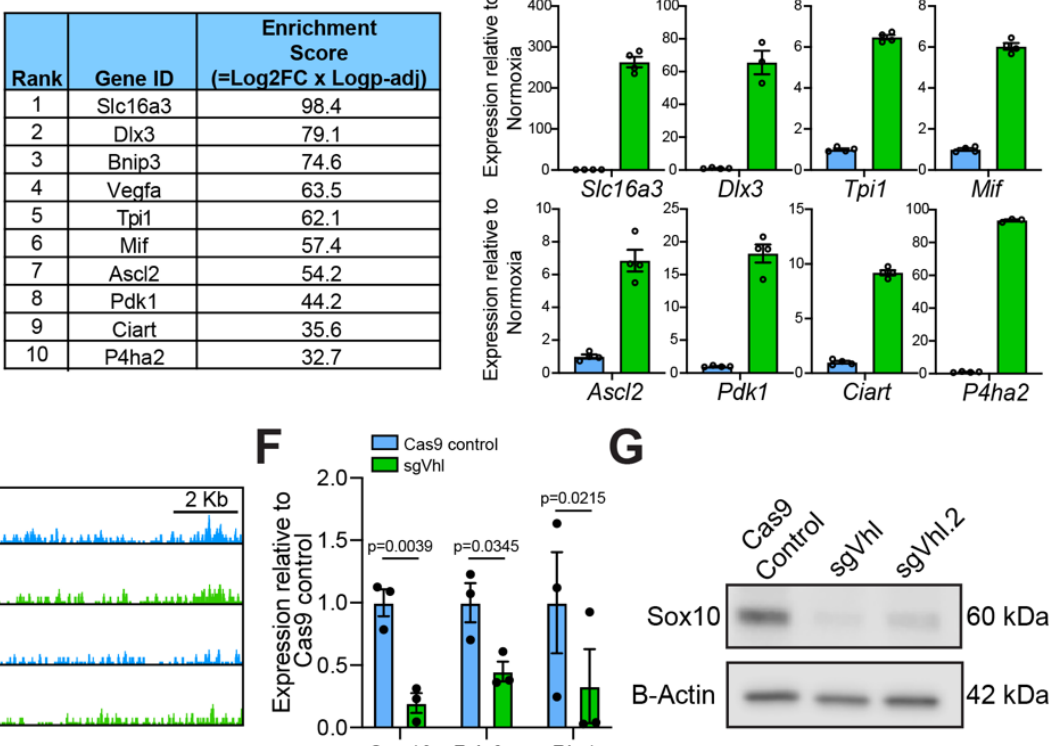

\section{Figure 2. HIF1a Accumulation Suppresses Sox10 Expression}

(A) Aggregate binding profile and heatmap of the 503 HIF1a peaks called by MACS2 (narrow peaks, FDR $<0.001$ ) in sgVhl OPCs within $2 \mathrm{~Kb}$ of the transcription start site (TSS) and transcription end site (TES) of the closest expressed gene in both Cas9 control and sgVhl OPCs normalized to input.

(B) Volcano plot of genes that significantly increase (in yellow) and decrease (in blue) with direct targets of HIF1a in black in sgVhl compared to Cas9 control OPCs (p-adj <0.05). Gray dots are genes not significantly different between conditions. Genes indicated in italics represent examples of top direct HIF1a targets in OPCs. Data are from 3 biological replicates (independent samples). 
(C) Venn diagram highlighting top functional HIF1a targets in OPCs by overlapping genes with proximal HIF1a binding (FDR $<0.001$, in green), significant gains in H3K27ac (FDR $<0.1$, in blue) and significantly increased expression ( $P$-adj $<0.05$, in purple). Genes were then ranked by their enrichment score, which is the product of the $\log _{2}$ (Fold change in gene expression) and -Log(Padj). The top 10 targets are displayed in the table ranked by their respective enrichment scores.

(D) qRT-PCR of 8 top HIF1a target genes (see Figure 1D for Vegfa and Bnip3) in OPCs treated with hypoxia $\left(1 \% \mathrm{O}_{2}\right.$, in green) compared to normoxia $\left(21 \% \mathrm{O}_{2}\right.$, in blue) normalized to endogenous loading control Rp/13a. Data are presented as mean \pm SEM from 3-4 technical replicates (individual wells) per condition.

(E) Genome browser view of H3K27ac and HIF1a signals in Cas9 control (in blue) and sgVhl (in green) OPCs normalized to input at the locus for Sox10 with a reduction in H3K27ac proximal to the Sox10 promoter highlighted in gray. Scale bar, $2 \mathrm{~Kb}$.

(F) qRT-PCR of Sox10 and downstream Sox10 target genes Pdgfra and Plp1 in sgVhl OPCs (in green) compared to Cas9 control OPCs (in blue) normalized to endogenous loading control Rpl13a. Data are presented as mean \pm SEM from 3 biological replicates (independent experiments) with 3-4 technical replicates (individual wells) per experiment. $p$-values were calculated using Student's two-tailed t-test.

(G) Western blot of nuclear lysates for SOX10 in sgVhl and Vhl.2 OPCs compared to Cas 9 control OPCs relative to B-Actin loading control. Molecular weight is indicated to the right of the blot.

See also Figure S2. 
A

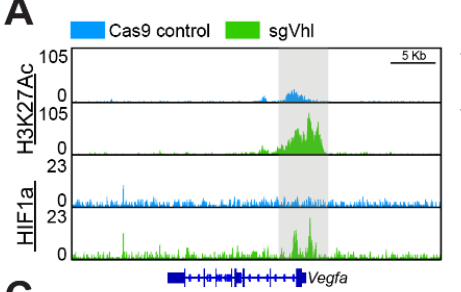

C Cas9 control sgVhl
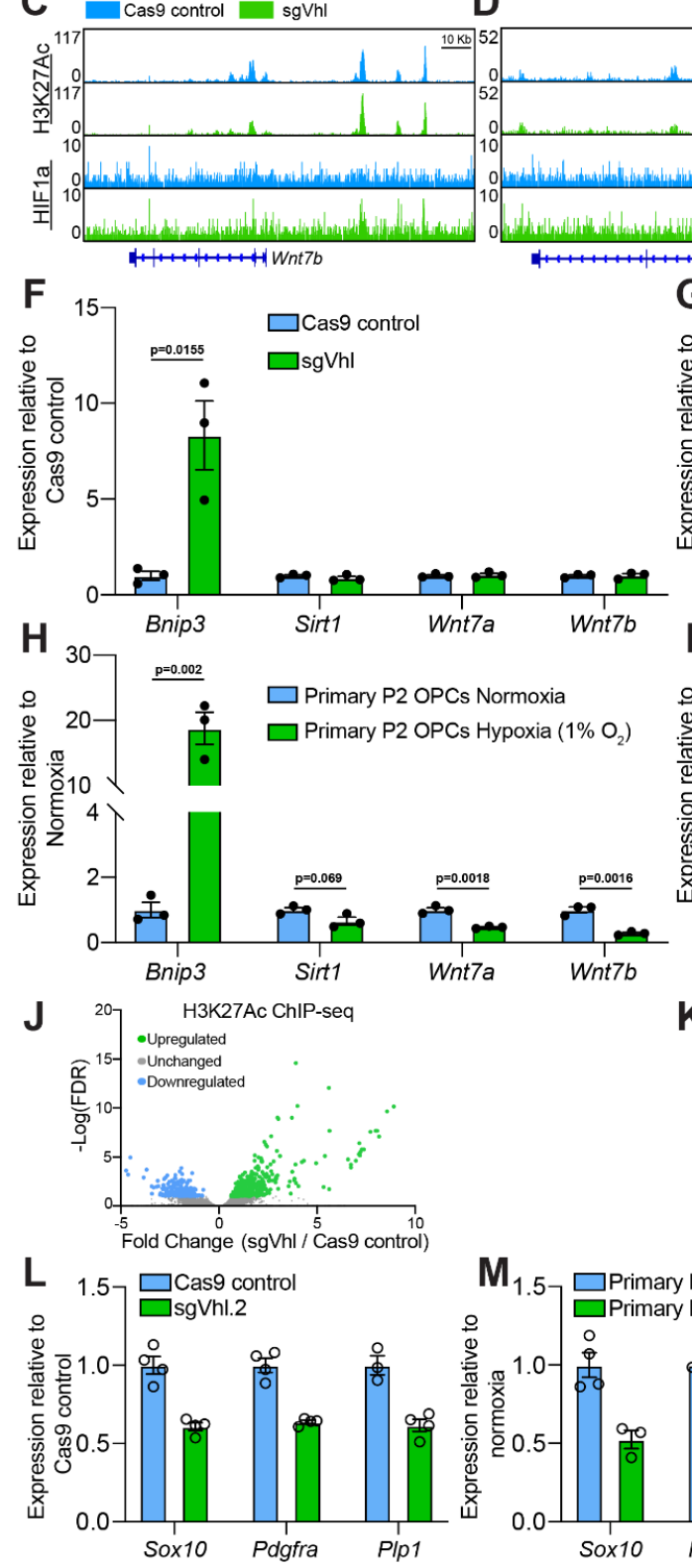

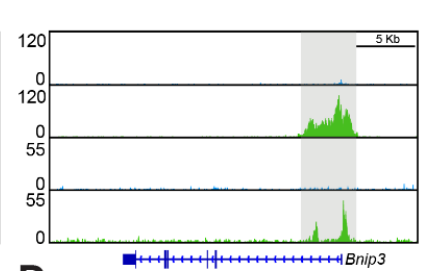

D

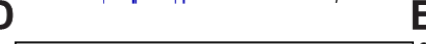

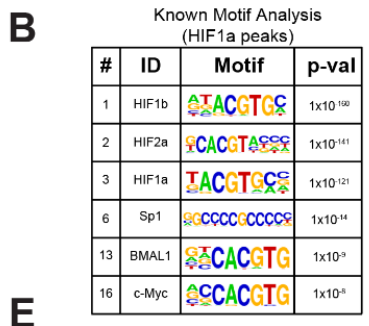
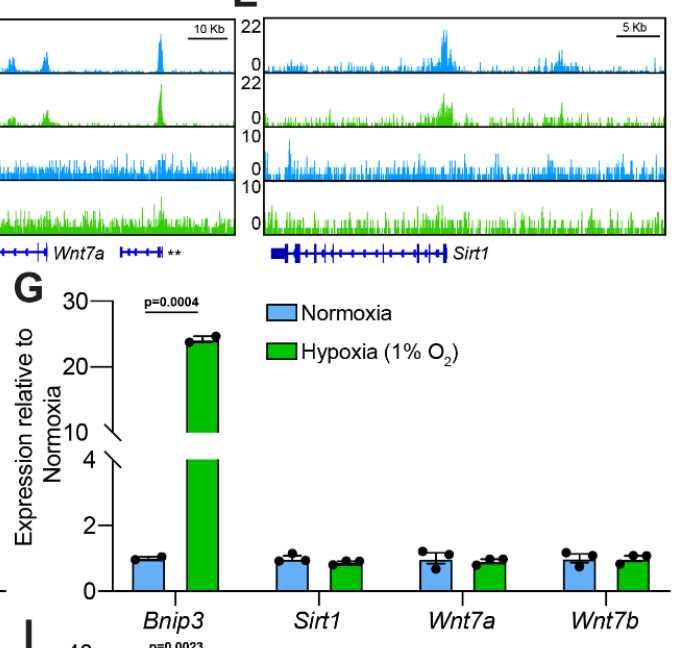

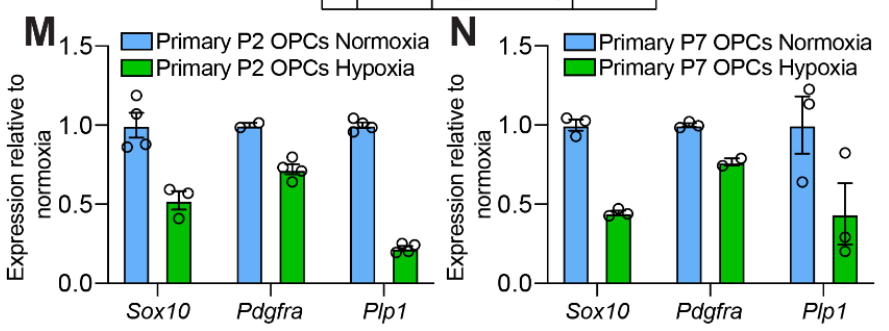

Figure S2. HIF1a Accumulation Suppresses Sox10 Expression, Related to Figure 2.

(A) Genome browser view of H3K27ac and HIF1a signals in Cas9 control (in blue) and sgVhl (in green) OPCs normalized to input at the locus for Vegfa and Bnip3. HIF1a and active chromatin 995 accumulation in sgVhl OPCs are highlighted in gray. Scale bars, $5 \mathrm{~Kb}$. 
(B) Table of known motifs significantly enriched in HIF1a peaks in sgVhl OPCs (FDR<0.001) displaying the transcription factor name, motif, and p-value ranked in order of significance (\# indicates rank out of all motifs from the analysis).

(C-E) H3K27ac and HIF1a ChIP-seq in Cas9 control (in blue) and sgVhl (in green) OPCs normalized to input at the locus for Wnt7b. Scale bars, $10 \mathrm{~Kb}(\mathrm{C}-\mathrm{D})$ and $5 \mathrm{~Kb}(\mathrm{E})$. $^{* *}$ is 4930471M09Rik.

(F-I) qRT-PCR of Bnip3, Sirt1, Wnt7a or Wnt7b in Cas9 control OPCs (in blue) and sgVhl OPCs (in green) $(F)$, OPCs treated with normoxia (in blue) or hypoxia $\left(1 \% \mathrm{O}_{2}\right.$, in green) $(\mathrm{G})$, primary postnatal day 2 (P2) in vivo derived mouse OPCs cultured in physiological hypoxia $\left(1 \% \mathrm{O}_{2}\right.$, in green) compared to normoxia (in blue) $(\mathrm{H})$, and primary immunopanned postnatal day 7 (P7) in vivo derived mouse OPCs cultured in physiological hypoxia $\left(1 \% \mathrm{O}_{2}\right.$, in green) compared to normoxia (in blue) (I) normalized to endogenous loading control Rp/13a. Data are presented as mean \pm SEM from 3 independent biological replicates (experiments) with 4 technical replicates (individual wells) per experiment. p-values were calculated using Student's two-tailed t-test.J) Volcano plot of fold change in intensity of H3K27ac peaks (FDR<0.001) between Cas9 control and sgVhl OPCs showing regions of significantly increased H3K27ac (FDR $<0.1$, in green) and decreased H3K27ac (FDR<0.1, in blue).

(J) Volcano plot of fold change in intensity of significant H3K27ac regions (FDR $<0.001)$ between Cas9 control and sgVhl OPCs showing regions of significantly increased H3K27ac (FDR<0.1, in green) and decreased H3K27ac (FDR<0.1, in blue).

(K) Table of known motifs in regions significantly enriched for H3K27ac (FDR<0.1) in sgVhl OPCs compared to Cas9 control OPCs displaying the transcription factor name, motif, and p-value ranked in order of significance (\# indicates rank out of all motifs from the analysis).

(L-N) qRT-PCR of Sox10 and downstream Sox10 target genes Pdgfra and Plp1 in sgVhl.2 (in green) compared to Cas9 control OPCs (in blue) (L), in primary postnatal day 2 (P2) in vivo derived mouse OPCs treated with physiological hypoxia (in green) compared to normoxia (in blue) (M), and in primary immunopanned postnatal day 7 (P7) in vivo derived mouse OPCs treated with physiological hypoxia (in green) compared to normoxia (in blue) (N). All qRT-PCRs are normalized to endogenous loading control Rp/13a. Data are presented as mean \pm SEM from 3-4 technical replicates (individual wells) per condition. 
bioRxiv preprint doi: https://doi.org/10.1101/2020.04.03.003632; this version posted April 16, 2020. The copyright holder for this preprint (which was not certified by peer review) is the author/funder, who has granted bioRxiv a license to display the preprint in perpetuity. It is made available under aCC-BY-NC-ND 4.0 International license.

A

A HIF1a gene targets in

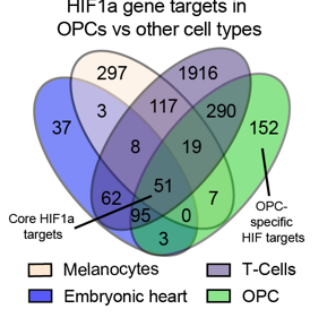

D
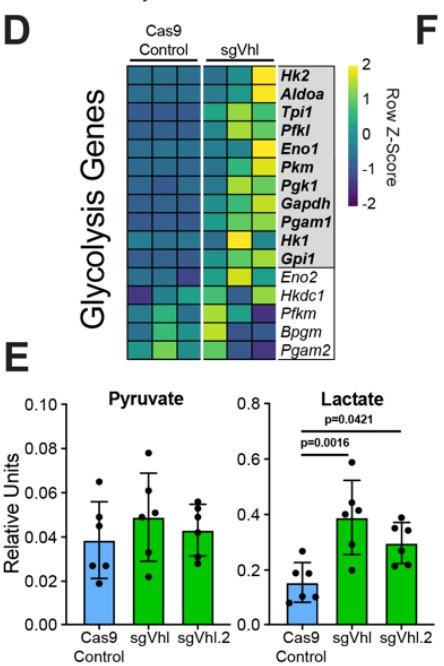

G $\square$ Cas9 control OPCs $\square$ sgVhl OPCs $\square$ Embryonic heart $\square$ Melanocytes $\square$ T-Cells

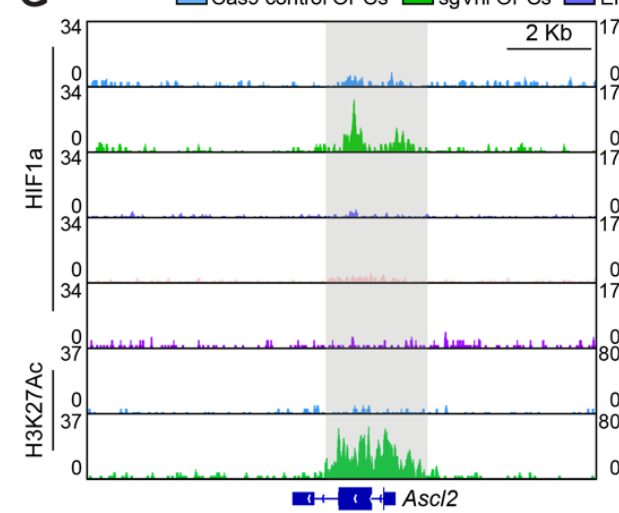

F HIF1a
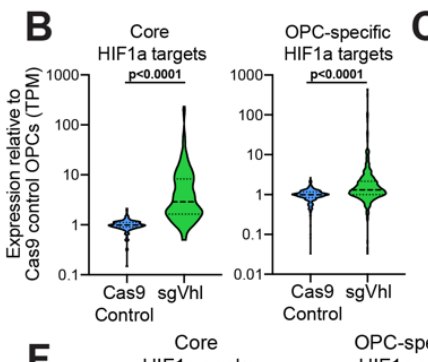

C
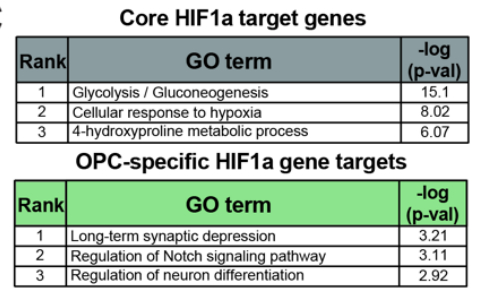

Control sgit

OPC-specific
HIF1a peaks

Other tissue specific
HIF1a peaks

Control sgVhl
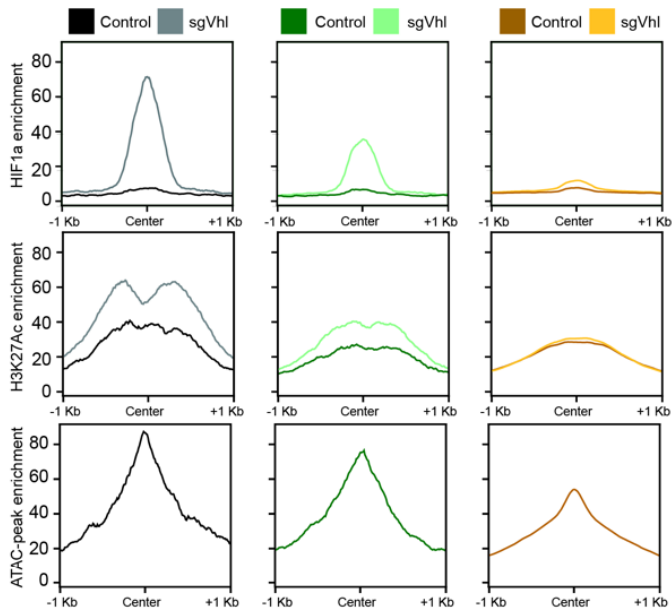

${ }^{-1 \mathrm{~Kb} \quad \text { Center }}$
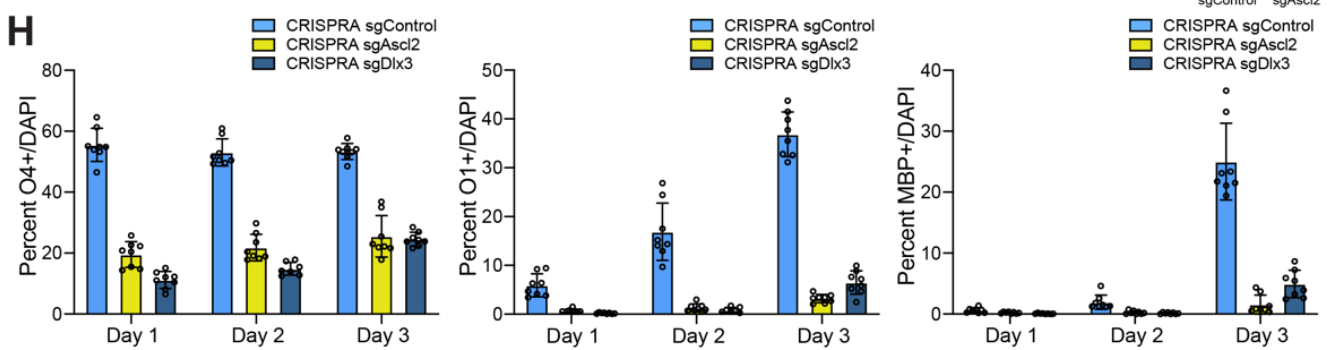
(A) Venn diagram of direct HIF1a target genes from publicly available HIF1a ChIP-seq datasets in diverse mouse tissues overlapped with HIF1a targets in OPCs (using MACS2, narrowpeaks, FDR $<0.001$, limited to genes within $5 \mathrm{~Kb}$ of significant HIF1a peaks).

(B) Violin plots of compiled expression data (TPM) normalized to Cas9 control OPCs for genes in core and OPC-specific HIF1a target categories in Cas9 control (in blue) and sgVhl (in green). Bold dashed line represents the median with the thin dashed lines representing the upper and lower quartiles. p-values were calculated using Mann Whitney two-tailed t-test.

(C) Gene ontology (GO) analysis of genes that are core targets of HIF1a in all 4 tissue types as well as genes that are targets of HIF1a specifically in OPCs. Table shows the rank of the GO term along with -log(p-value).

(D) Heatmap of row normalized expression of genes in the glycolysis pathway between Cas9 control and sgVhl OPCs. Genes with their names bolded and in the gray box are direct targets of HIF1a and exhibit increased expression in OPCs.

(E) Targeted metabolomics for pyruvate and lactate in both sgVhl and sgVhl.2 OPCs (both in green) compared to Cas 9 control OPCs (in blue). Data are presented in relative units as mean \pm SD from 6 biological replicates (independent experiments) with one technical replicate (individual well) per experiment. p-values were calculated using one-way ANOVA with Dunnett's multiple comparisons test.

(F) Aggregate plots of HIF1a and H3K27ac enrichment in Cas9 control and sgVhl OPCs as well as open chromatin (defined by ATAC-seq) enrichment in normal, non-transduced OPCs at core HIF1a peaks shared by all 4 tissue types, OPC-specific HIF1a peaks, and other tissue-specific HIF1a peaks (combination of cell-type-specific peaks from heart, T-cells, and melanocytes).

(G) Genome browser view showing HIF1a enrichment at Asc/2 and DIx3 loci in sgVhl OPCs (in green) compared to Cas9 control OPCs (in blue), embryonic heart (in light purple), melanocytes (in beige) and T-cells (in magenta) normalized to input. H3K27ac enrichment is also shown in sgVhl OPCs (in green) compared to Cas9 control OPCs (in blue). HIF1a and H3K27ac accumulation in sgVhl OPCs are highlighted in gray (Scale bars are $2 \mathrm{~Kb}$ ).

(H) Quantification of the percentage of early $\mathrm{O}^{+}+$, intermediate $\mathrm{O} 1+$, and late $\mathrm{MBP}+$ oligodendrocytes in both sgAscl2 (in yellow) and sgDIx3 (in dark blue) CRISPRA OPCs compared to CRISPRA sgControl (in light blue) OPCs at day 1,2 and 3 of differentiation. Data are presented as mean \pm SD of 6-8 technical replicates (individual wells) per condition.

(I) qRT-PCR of Sox10 in CRISPRA sgAscl2 (in yellow) and CRISPRA sgDIx3 (in dark blue) compared to CRISPRA sgControl OPCs normalized to endogenous loading control Rp/13a. Data are presented as mean \pm SEM from 3 biological replicates (independent experiments) with 3-4 technical replicates (individual wells) per experiment. p-values were calculated using Student's two-tailed t-test.

See also Figure S3. 
A

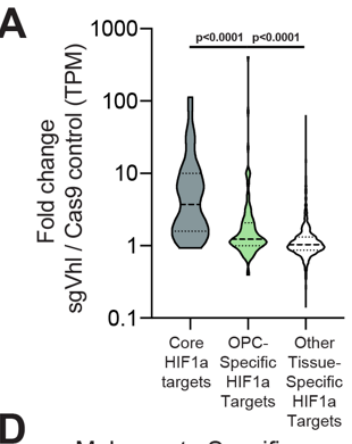

D

\begin{tabular}{|c|c|c|c|}
\hline \multicolumn{4}{|c|}{ Melanocyte Specific } \\
\hline \# & ID & Motif & p-val \\
\hline 1 & Rfx3 & GSOTICCCATGCAAC & $1 \times 10^{-148}$ \\
\hline 8 & Tfe3 & ETCACGTGACCC & $1 \times 10^{-42}$ \\
\hline 13 & Mitf & SICATGTGC & $1 \times 10^{-23}$ \\
\hline
\end{tabular}

E

scRNA-seq "Brain Non-myeloid" (Tabula Muris)

\begin{tabular}{|c|c|c|c|c|c|c|}
\hline Gene & Pericyte & Astrocyte & OPC & Oligodendrocyte & Neuron & $\begin{array}{c}\text { Endothelial } \\
\text { Cell }\end{array}$ \\
\hline Olig2 & 0.05 & 0.03 & 6.80 & 4.01 & 0.31 & 0.14 \\
\hline SIc16a3 & 0.29 & 0.04 & 0.12 & 0.32 & 0.18 & 0.14 \\
\hline$D 1 \times 3$ & 0.02 & 0.01 & 0.00 & 0.00 & 0.00 & 0.00 \\
\hline Ascl2 & 0.01 & 0.01 & 0.00 & 0.01 & 0.03 & 0.03 \\
\hline
\end{tabular}

G

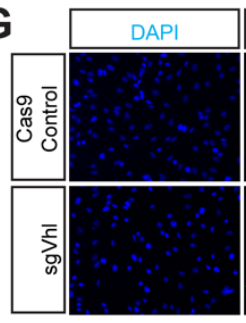

I
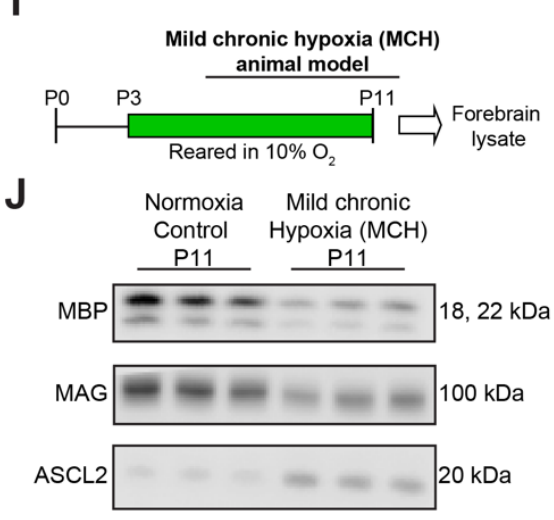

B-Actin $\square 42 \mathrm{kDa}$
B Melanocyte specific HIF1a gene targets

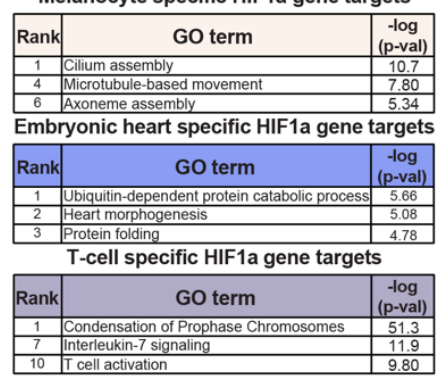

C

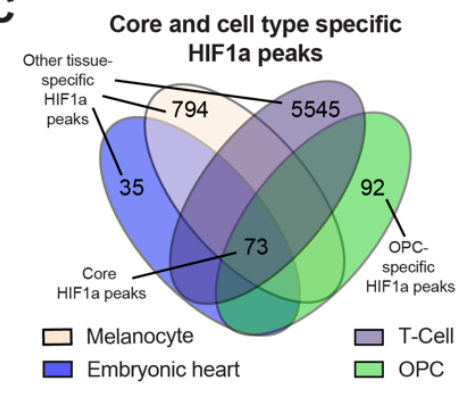

T-cell Specific*

\begin{tabular}{|c|c|c|c|}
\hline$\#$ & ID & Motif & p-val \\
\hline 1 & Batf & TATGACTCAT & $1 \times 10^{-210}$ \\
\hline 2 & Atf3 & SATGAGTCACEC & $1 \times 10^{-208}$ \\
\hline 3 & Junb & SATGAGTCAS & $1 \times 10^{-208}$ \\
\hline
\end{tabular}

\begin{tabular}{|c|c|c|c|}
\multicolumn{4}{c|}{ OPC-Specific* } \\
\hline$\#$ & ID & Motif & p-val \\
\hline 1 & Bhlhe40 & ICACGTCCCE & $1 \times 10^{-4}$ \\
\hline 2 & Bhlhe41 & ICACGTGACC & $1 \times 10^{-3}$ \\
\hline 23 & Olig2 & CCATCTTCT: & $1 \times 10^{-1}$ \\
\hline
\end{tabular}

$\mathbf{F}$

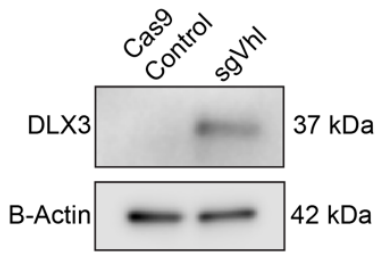

H $\square$ Normoxia $\square$ Hypoxia $\left(1 \% \mathrm{O}_{2}\right)$
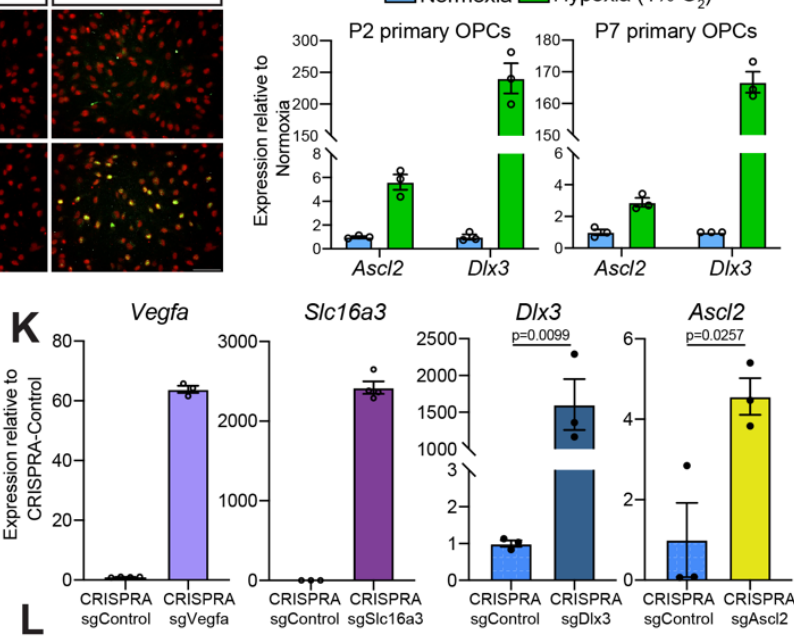

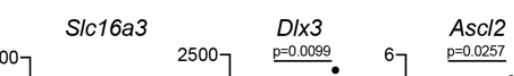

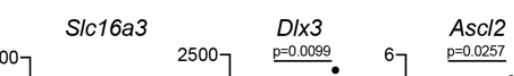

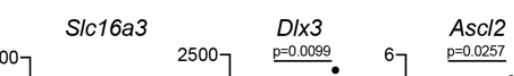

ASCL2 OLIG2

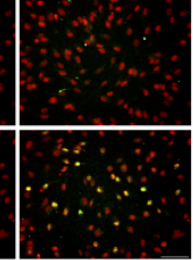

sgControl sgVegfa sgControl sgSlc16a3

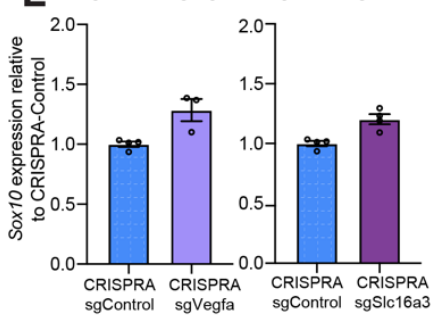

Figure S3. OPC-specific HIF1a Targets Downregulate Sox10, Related to Figure 3.

(A) Violin plot of fold change in expression (TPM) between Cas9 control and sgVhl OPCs of genes included in core (in dark gray), OPC-specific (light green) and other tissue-specific (light gray) 
HIF1a target categories. Bold dashed line represents the median with the thin dashed lines representing the upper and lower quartiles. p-values were calculated using the Kruskal Wallis One-Way ANOVA with Dunn's multiple comparisons test.

(B) Gene ontology (GO) analysis of genes targeted by HIF1a specifically in melanocytes (beige), embryonic heart (purple), and T-cells (magenta). The chart includes curated pathways with their rank based on their respective $p$-values.

(C) Overlap of significant HIF1a peaks (FDR<0.001) across 4 different cell types giving core HIF1a peaks, OPC-specific HIF1a peaks and other tissue-specific HIF1a peaks (combination of heart, T-cell and melanocyte specific peaks).

(D) Table of known motifs significantly enriched in cell-type-specific HIF1a peaks in melanocytes (beige), heart (purple), T-cells (magenta) and OPCs (light green). Charts display the transcription factor name, motif, and $p$-value ranked in order of significance (\# indicates rank out of all 440 motifs in the analysis and * indicates that HIF motifs were removed).

(E) Chart of publicly available single-cell RNA-seq data from non-myeloid cells of the brain for expression of DIx3, Ascl2 and S/c16a3 with positive control Olig2, which marks OPCs and oligodendrocytes. Values are $\ln (1+\mathrm{CPM})$. CPM is counts per million reads.

(F) Western blot of nuclear lysates for DLX3 in sgVhl OPCs and CRISPR-control OPCs with BActin as the loading control. Molecular weight is indicated to the right of the blot.

(G) Immunocytochemistry (ICC) for ASCL2 (in green) and OLIG2 (in red) in Cas9 control and sgVhl OPCs. Nuclei are marked by DAPI (in blue).

(H) qRT-PCR of D/x3 and Ascl2 in P2 and P7 primary in vivo derived OPCs treated with physiological hypoxia $\left(1 \% \mathrm{O}_{2}\right.$, in green) compared to normoxia $\left(21 \% \mathrm{O}_{2}\right.$, in blue) normalized to endogenous loading control Rp/13a. Data are presented as mean \pm SEM from 3 technical replicates (individual wells) per condition.

(I) Schematic highlighting the mild chronic hypoxia model of diffuse white matter injury in which mouse pups are reared in 10\% oxygen from postnatal day 3 to 11 (P3 to P11) to model diffuse white matter injury of prematurity. Animals are sacrificed at postnatal day (P11) and their forebrains are isolated and lysed for protein.

(J) Western blots of forebrain lysates from P11 female C57BI6 mice of myelin proteins MBP and MAG along with ASCL2 in animals reared in hypoxia from P3-P11 compared to control normoxic reared mice. B-Actin is shown as a loading control. $\mathrm{N}=3$ female C57BI6 mice per treatment group. Molecular weight is indicated to the right of the blot.

(K) qRT-PCR of Vegfa, S/c16a3, Ascl2 and D/x3 normalized to endogenous loading control $R p / 13 a$ in their respective CRISPRA OPCs compared to CRISPRA sgControl OPCs. Data are presented as mean \pm SEM from 3-4 technical replicates (individual wells) per condition.

(L) qRT-PCR of Sox10 in CRISPRA sgVegfa (light purple) and CRISPRA sgSlc16a3 (in magenta), OPCs compared to CRISPRA sgControl OPCs normalized to endogenous loading control Rp/13a. Data for CRISPRA sgVegfa and CRISPRA sgSlc16a3 are presented as mean \pm SEM from 3-4 technical replicates (individual wells) per condition. 
FIGURE 4
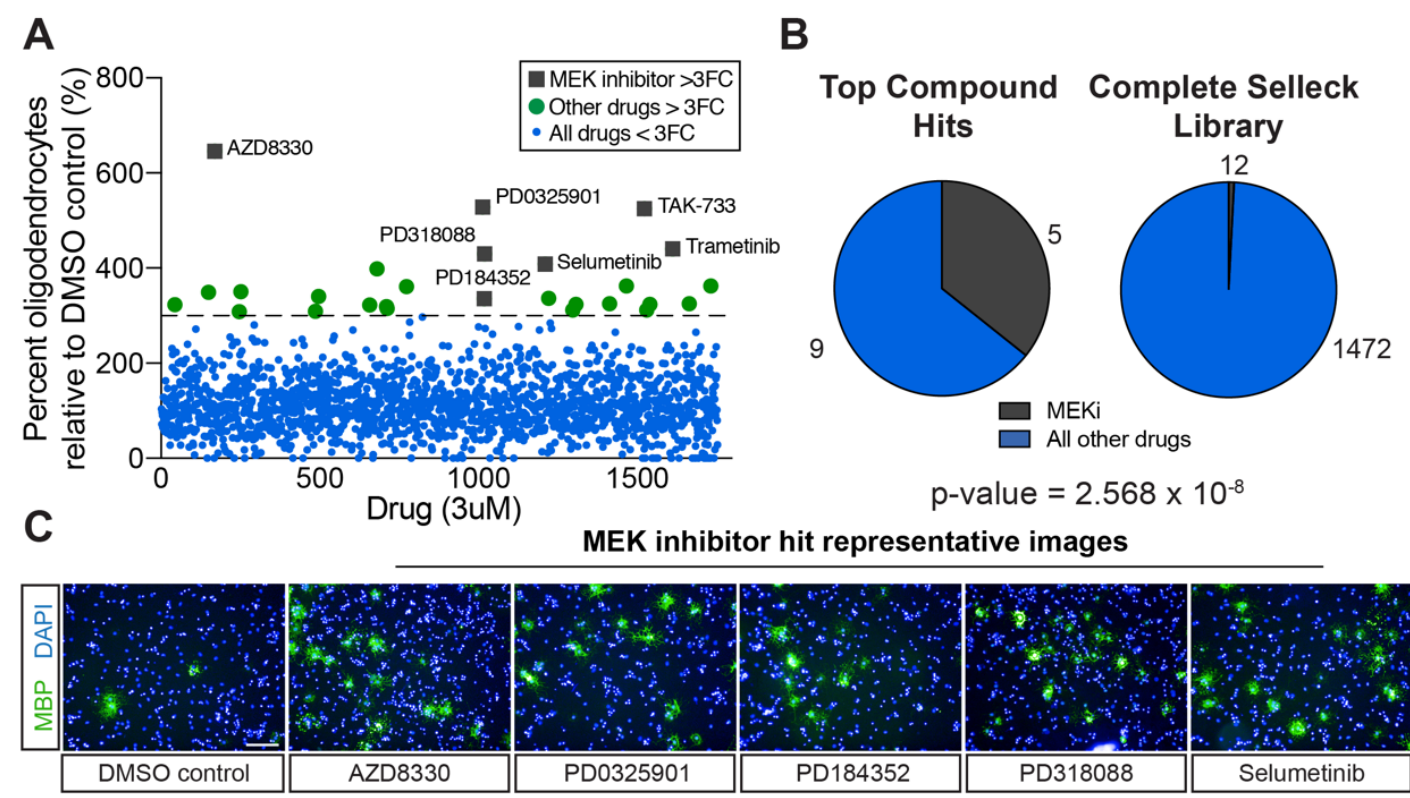

D

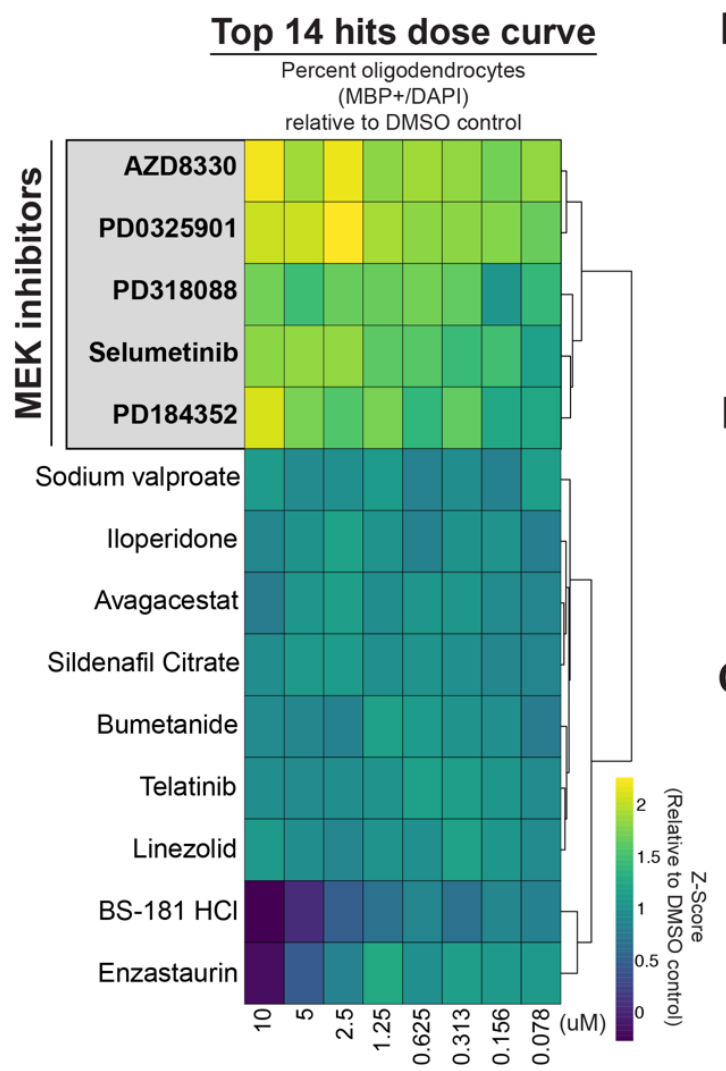

E

$\mathbf{F}$

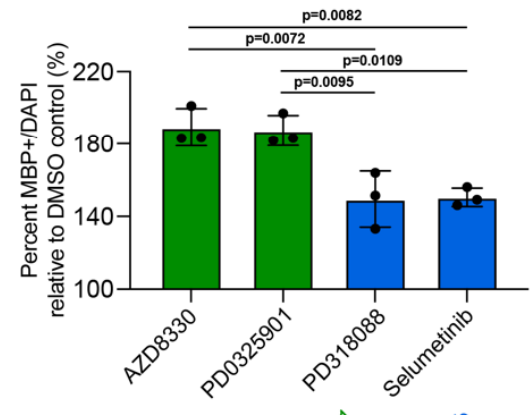

F

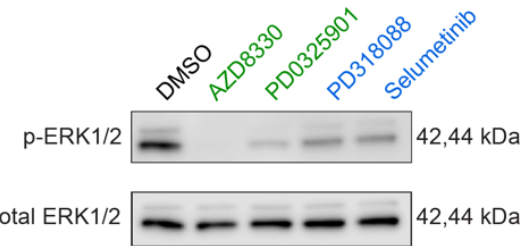

G

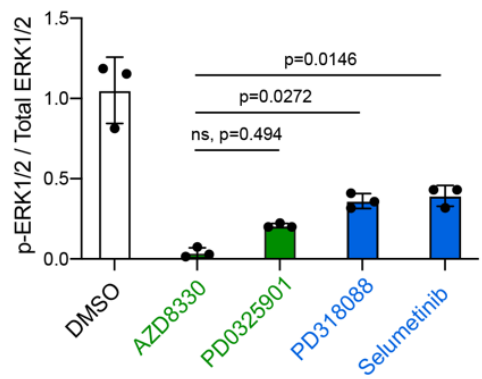

(A) Primary bioactives library screen showing the effect of 1753 molecules on percentage of oligodendrocytes (MBP+ cells/ total DAPI) formed by sgVhl OPCs relative to DMSO treated sgVhl 
OPCs. Anything above the dotted line represents a greater than 3-fold change increase in percentage of oligodendrocytes from DMSO (green dots). MEK inhibitors are highlighted as gray boxes with their respective drug names.

(B) Pie charts of the number of non-toxic MEK inhibitors (in dark gray) compared to other classes of drugs (in blue) within top compound hits compared to their prevalence in the non-toxic compounds of the Selleck library. p-value was calculated using hypergeometric analysis.

(C) Representative ICC images of oligodendrocytes (MBP+ in green) from the primary drug screen of the 5 top MEK inhibitor hits along with the DMSO negative control. Nuclei are marked by DAPI (in blue). Scale bars, $100 \mu \mathrm{m}$.

(D) Heatmap representation of the top 14 hits in an 8-point dose curve in 2-fold dilutions from $10 \mu \mathrm{M}$ to $78 \mathrm{nM}$ showing the fold change in the percentage (MBP+/total DAPI) of oligodendrocytes relative to DMSO treated sgVhl OPCs. The heatmap is shown as row Z-score, and rows are sorted by unsupervised hierarchical clustering with columns in order from high $(10 \mu \mathrm{M})$ to low dose $(78 \mathrm{nM})$. MEK inhibitors are highlighted in gray and bolded. Data are presented as the mean for each drug at each dose from 3 separate dose curve plates.

(E) Collapsing all tested doses into one overall average shows the ability of each MEK inhibitor to increase the formation of oligodendrocytes (MBP+/DAPI) relative to DMSO treated sgVhl OPCs. AZD8330 and PD0325901 are shown in green representing the most effective drugs, while PD318088 and Selumetinib are shown in blue as slightly less effective drugs. Data are presented as the mean $\pm S D$ of the averages of all 8 doses for each drug from 3 separate dose curve plates. p-values were calculated using a one-way ANOVA with Tukey's multiple comparisons test.

(F) Representative Western blot for phosphorylated ERK1/2 (p-ERK1/2) relative to total ERK1/2 from whole cell lysates of sgVhl OPCs incubated with 100nM of AZD8330, PD0325901, PD318088 or Selumetinib for 30 minutes. Molecular weight is indicated to the right of the blot.

(G) Quantification of the ratio of phosphorylated ERK1/2 relative to total ERK1/2 for AZD8330 and PD0325901 (both in green) and PD318088 and Selumetinib (both in blue). Data are presented as mean \pm SD from 3 biological replicates (independent experiments) with a single technical replicate per experiment. $p$-values were calculated using one-way ANOVA with Dunnett's multiple comparisons test.

See also Figure S4. 
A

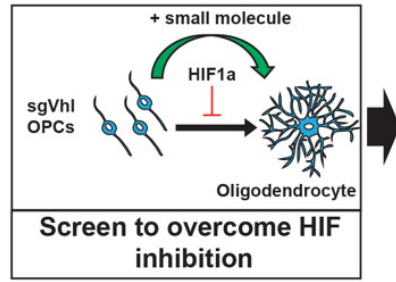

B

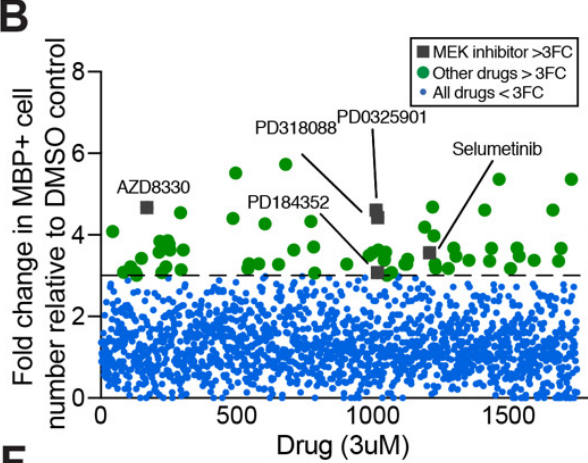

$\mathbf{F}$

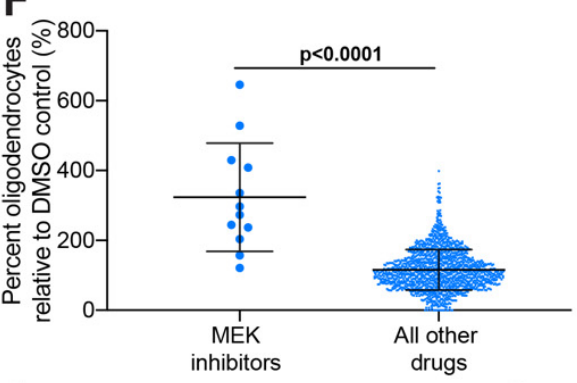

I

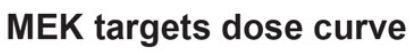

\begin{tabular}{|c|c|}
\hline Compound & Target \\
\hline Dinaciclib & Cdk \\
\hline MK-2206 2HCl & Akt \\
\hline CHIR-98014 & GSK-3 \\
\hline C188-9 & Stat3 \\
\hline Varespladib & Pla \\
\hline $10058-F 4$ & C-Myc \\
\hline CHIR-99021 & Gsk-3 \\
\hline Palbociclib & Cdk \\
\hline PF-4708671 & S6 Kinase \\
\hline AZD0364 & Erk1/2 \\
\hline Vx-11e & Erk1/2 \\
\hline SCH772984 & Erk1/2 \\
\hline &
\end{tabular}

$\mathbf{J}$

\section{G}

D

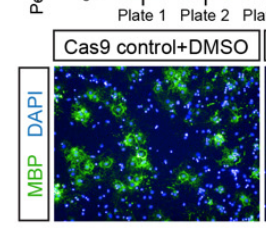

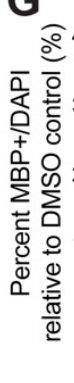

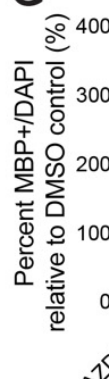

$\mathrm{sgVhl.2}$

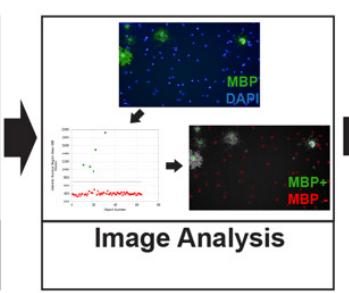

C

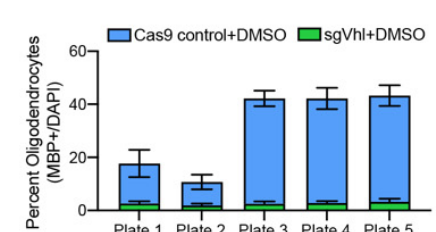
$\mathrm{H}$

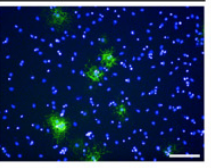

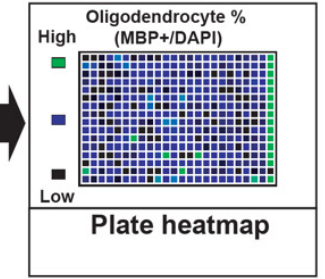

E

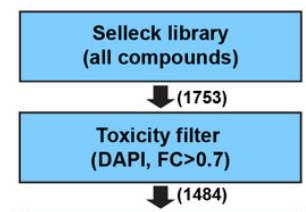

Increased OLs $(\mathrm{MBP}+\&$ MBP+/DAPI, $\mathrm{FC}>3)$

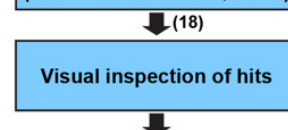

14 top hits from primary screen

\begin{tabular}{|c|c|c|}
\hline Compound & MEK1(IC50) & MEK2(IC50) \\
\hline${ }^{*}$ AZD8330 & $0.4 \mathrm{nM}$ & $0.4 \mathrm{nM}$ \\
\hline PD0325901 & $0.33 \mathrm{nM}$ & $0.33 \mathrm{nM}$ \\
\hline PD184352 & $17 \mathrm{nM}$ & $17 \mathrm{nM}$ \\
\hline PD318088 & unknown & unknown \\
\hline Selumetinib & $14 \mathrm{nM}$ & $530 \mathrm{nM}$ \\
\hline
\end{tabular}
"AZD8330 has IC50 for MEK1/2 of 7nM but has subnanomolar
potency in p-ERK functional assays (Selleck Chemicals)
potency in p-ERK functional assays (Selleck Chemicals)

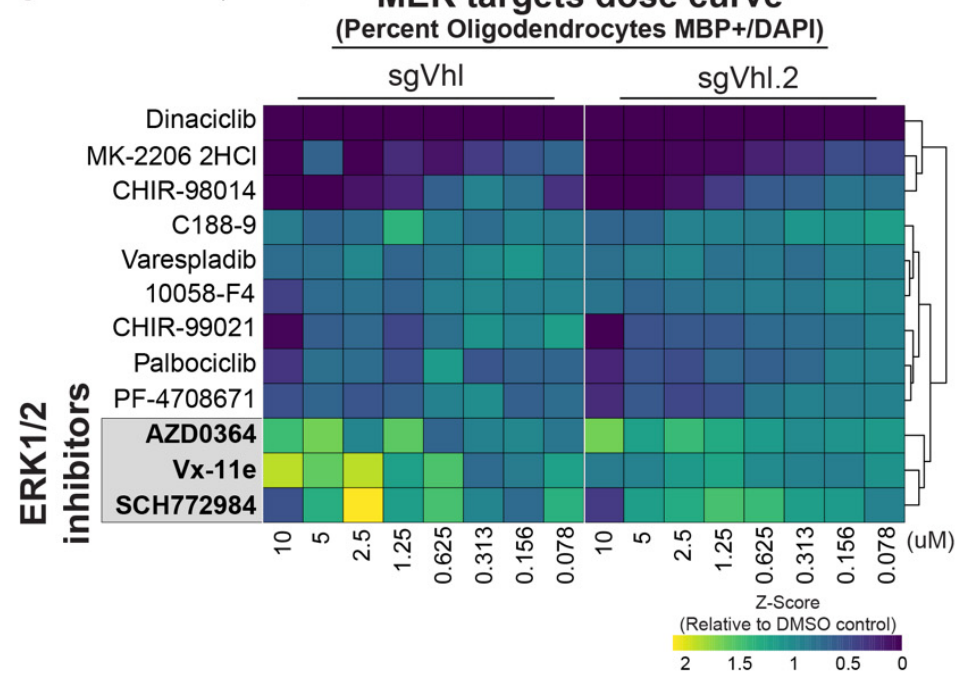

MEK targets dose curve Percent Oligodendrocytes MBP+/DAPI)

Figure S4. Inhibition of MEK/ERK Signaling Increases Oligodendrocyte Formation from sgVhl OPCs, Related to Figure 4.

(A) Schematic depicting the procedure for the primary bioactives screen to uncover compounds that increase oligodendrocyte formation from sgVhl OPCs. 
(B) Primary bioactives library screen showing the effect of 1753 small molecules on number of oligodendrocytes (MBP+ cells) formed by sgVhl OPCs relative to DMSO treated sgVhl OPCs. Anything higher than the dotted line represents a greater than 3-fold change increase from DMSO (green dots). MEK inhibitors are highlighted as gray boxes with their respective drug names.

(C) Primary screen positive control (Cas9 control+DMSO) and negative control (sgVhl+DMSO) percent oligodendrocyte $(\mathrm{MBP}+/ \mathrm{DAPI})$ metrics on a per plate basis. Data represent mean $\pm \mathrm{SD}$ from 16 technical replicates (individual wells) per plate per condition.

(D) Representative immunocytochemistry images of oligodendrocytes (MBP+ in green) from primary screen positive (Cas9 control+DMSO) and negative (sgVhl+DMSO) controls. Nuclei are marked by DAPI (in blue). Scale bars, $100 \mu \mathrm{m}$.

(E) Schematic detailing the filtering steps starting with the bioactives library and narrowing down to top hits that are non-toxic (total DAPI FC<0.7), increased the number and percentage of oligodendrocytes ( $F C>3$ ) and passed visual inspection to give the top 14 compound hits. Numbers in parentheses represent the number of drugs after each filtering step.

(F) Quantification of the effect of all non-toxic MEK inhibitors ( $n=12$ ) compared to all other nontoxic drugs from the primary screen $(n=1472)$ on the percentage of oligodendrocytes from sgVhl OPCs relative to DMSO treated sgVhl OPCs. Data are presented as mean \pm SD. $p$-values were calculated using the Mann-Whitney t-test.

(G) Collapsing all tested doses into one overall average shows the ability of each MEK inhibitor to increase the formation of oligodendrocytes (MBP+/DAPI) from sgVhl.2 OPCs relative to DMSO treatment. AZD8330 and PD0325901 are shown in green representing the most effective drugs, while PD318088 and Selumetinib are shown in blue as slightly less effective drugs. Data are presented as the mean $\pm \mathrm{SD}$ of all 8 doses for each drug from a single dose curve plate.

(H) Table of IC50 values for MEK1 and MEK2 for the top MEK inhibitors AZD8330, PD035901, PD184352, and Selumetinib. IC50 is still currently unknown for PD318088.

(I) List of the 12 drugs included on the MEK targets dose curve plate including the drug name and canonical target of the drug.

(J) Heatmap representation of the 8-point dose curve performed for all 12 drugs in the MEK target dose curve plate shown as row Z-score. Data in the heatmap is the fold change in percent oligodendrocytes (MBP+/total DAPI) in sgVhl and sgVhl.2 OPCs relative to their respective DMSO negative controls. Rows were sorted by unsupervised hierarchical clustering and columns are in order from high $(10 \mu \mathrm{M})$ to low dose $(78 \mathrm{nM})$ of drug. ERK1/2 inhibitors are highlighted in gray and bold. Data are presented as the mean for each drug at each dose from 2 separate dose curve plates for both sgVhl and sgVhl.2 OPCs. 
A

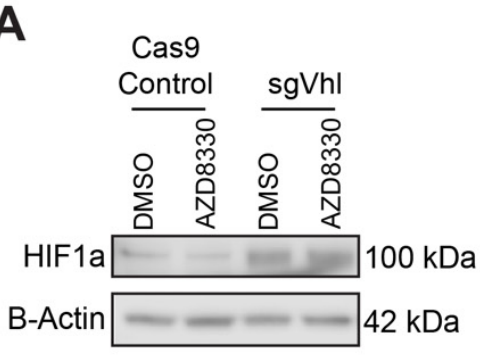

B

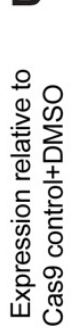

$\begin{aligned} & \text { C } \text { Cas9 control+DMso } \\ & \text { sgVhl+DMSO } \\ & \text { sgVhl +AZD8330 }\end{aligned}$

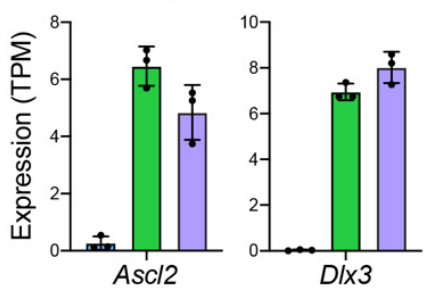

D Cas9 control+DMSO vs sgVhl+DMSO

E

GO: Oligodendrocyte Development

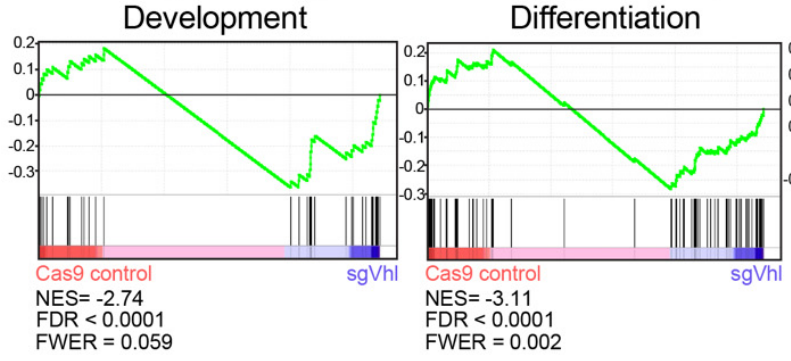

F

F GO: Oligodendrocyte Development

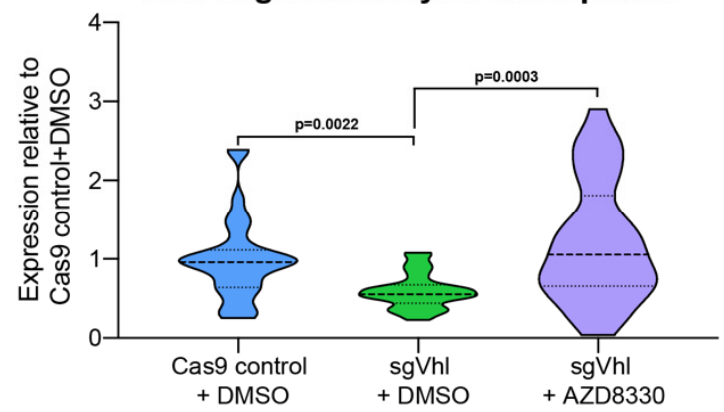

H

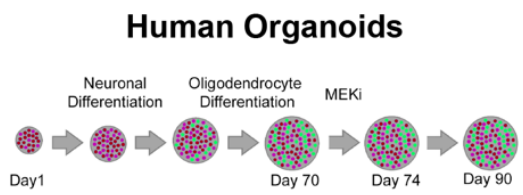

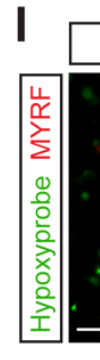

G
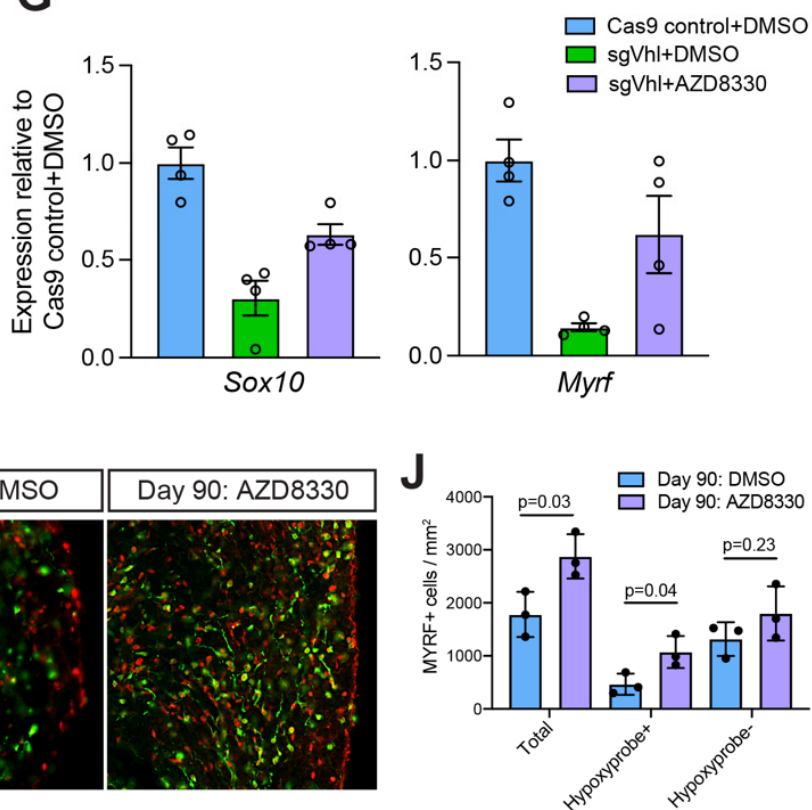

Figure 5. MEK Inhibitors Restore Sox10 Expression without Changing HIF1a activity in OPCs

(A) Western blot of nuclear lysates for HIF1a in sgVhl OPCs with 24hrs of 300nM AZD8330 treatment compared to DMSO with B-Actin as a loading control. Molecular weight is indicated to the right of the blot. 
(B) Violin plot of normalized number of transcripts (TPM values normalized to Cas9 control+DMSO) in Cas9 control+DMSO (in blue), sgVhl + DMSO (in green), and sgVhl + AZD8330 (in purple) relative to Cas9 control + DMSO of genes that were previously shown to be direct targets of HIF and increase in sgVhl OPCs compared to control (see Figure 2B). p-values were calculated using the Kruskal Wallis One-Way ANOVA with Dunn's multiple comparisons test.

(C) Quantification of the normalized number of transcripts (TPM) for both Asc/2 and D/x3 in Cas9 control+DMSO (in blue), sgVhl+DMSO OPCs (in green) and sgVhl+AZD8330 OPCs (in purple). OPCs were treated with either DMSO or 300nM AZD8330 for 14 hours. Data represent mean \pm $\mathrm{SD}$ from 3 biological replicates (independent samples) from RNA-seq.

(D) Gene set enrichment analysis (GSEA) analysis of gene program changes in sgVhl compared to Cas9 control OPCs demonstrates a significant reduction in GO terms for Oligodendrocyte Development (normalized enrichment score/NES $=-2.74$, FDR<0.0001, FWER p-val =0.059) and Oligodendrocyte Differentiation (NES = -3.11, FDR<0.0001, FWER p-val = 0.002).

(E) GSEA analysis of gene program changes in sgVhl + AZD8330 compared to sgVhl + DMSO OPCs demonstrates a significant enrichment in GO terms for Oligodendrocyte Development (normalized enrichment score/NES $=2.98, \mathrm{FDR}=5.5 \times 10^{-6}, \mathrm{FWER} \mathrm{p}$-val $=0.004$ ) and Oligodendrocyte Differentiation (NES $=3.58$, FDR<1 $1 \times 10^{-6}$, FWER p-val <0.001).

(F) Violin plot showing expression of transcripts (TPM values normalized to Cas9 control + DMSO OPCs) associated with GO term Oligodendrocyte Development (GO:0014003) that decrease $(\mathrm{FC}<0.75)$ in sgVhl + DMSO OPCs (in green) relative to Cas9 control + DMSO OPCs (in blue) as well as sgVhl OPCs following treatment with 300nM AZD8330. p-values were calculated using the Kruskal Wallis One-Way ANOVA with Dunn's multiple comparisons test.

(G) qRT-PCR of Sox10 and Myrf in Cas9 control+DMSO (in blue), sgVhl+DMSO (in green) and sgVhl+AZD8330 OPCs (in purple) normalized to endogenous control Rp/13a. OPCs were treated with either DMSO or 300nM AZD8330 for 14 hours. Data are presented as mean \pm SEM from 4 technical replicates (individual wells).

(H) Schematic of human brain oligocortical spheroids treated at days in vitro 70 with either DMSO or 300nM AZD8330 for 4 days. The spheroids were then cultured without drug until day 90 when they were incubated with hypoxyprobe, fixed and then sectioned for immunohistochemistry.

(I) Representative immunohistochemistry images of DIV 90 oligocortical spheroids that had been treated from DIV 70-74 with either DMSO or 300nM AZD8330 for oligodendrocytes (MYRF+ in red) and hypoxic regions (hypoxyprobe in green). Scale bar, 100 $\mathrm{MM}$.

(J) Quantification of oligodendrocytes (MYRF+ / $\mathrm{mm}^{2}$ ) in the whole oligocortical spheroid (total), hypoxic region of the spheroid (hypoxyprobe+), and normoxic region of the spheroid (hypoxyprobe-) in DIV 90 spheroids that had been treated with either DMSO or 300nM AZD8330 from DIV 70-74. Data represent mean \pm SD from 3 biological replicates (individual spheroids). $p$ values were calculated using Student's two-tailed t-test.

See also Figure S5. 


\section{FIGURE S5}
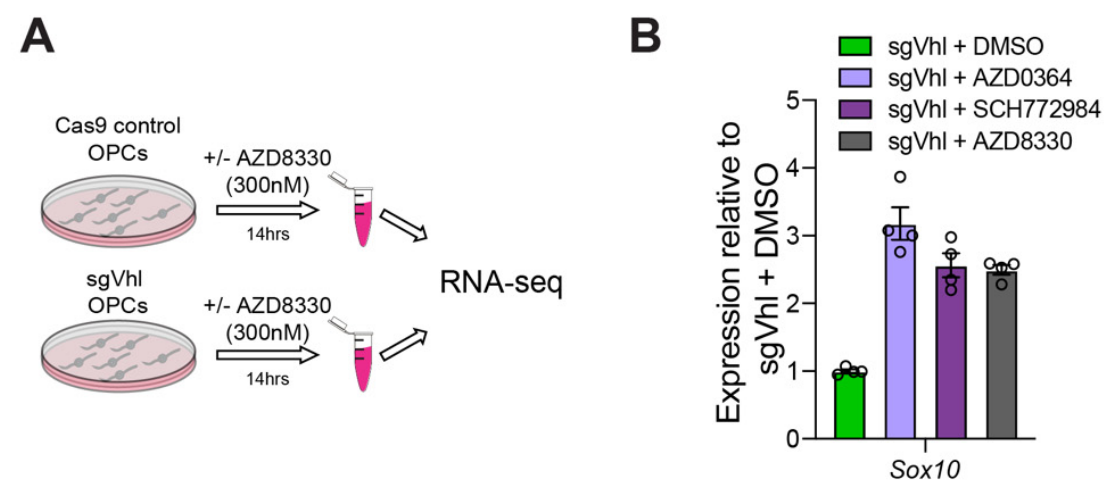

Figure S5. Inhibition of MEK/ERK Signaling Drives Sox10 Expression in sgVhl OPCs, Related to Figure 5.

(A) Schematic highlighting the setup of the RNA-seq experiment in which Cas9 control and sgVhl OPCs were treated with either DMSO or 300nM of AZD8330 for 14 hours and then cells were lysed for poly-adenylated mRNA extraction and sequenced.

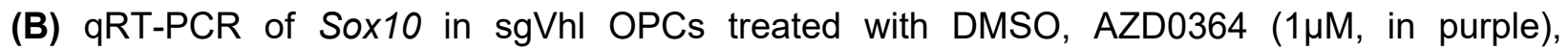

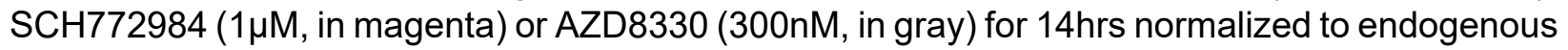
control $R p / 13 a$. Data are presented as mean \pm SEM from 4 technical replicates (individual wells) per condition. 